\title{
The Application of Metabolomic Profiling to the Effects of Physical Activity
}

\author{
Evangelia Daskalaki ${ }^{1}$, Chris Easton ${ }^{2}$ and David G. Watson ${ }^{1 *}$ \\ ${ }^{1}$ Strathclyde Institute of Pharmacy and Biomedical Sciences, University of Strathclyde, Glasgow, United \\ Kingdom; ${ }^{2}$ Institute for Clinical Exercise and Health Science, University of the West of Scotland
}

\begin{abstract}
Physical activity and exercise are potent lifestyle interventions for the reduction of several psycho-somatic risks, including cardiovascular disease, diabetes and mild-to-moderate depression. The current review describes the studies which have utilised a metabolomics/ metabolite profiling approach in combination with exercise in the period between 1999-2014 in predominantly healthy subjects (N:64) and in a smaller subset of patient cohorts $(\mathrm{N}: 10)$ between 2011-2014. A broad range of intervention types were used in both healthy and patient cohorts with varying exercise regimes with or without supplement use. Effects on purine metabolism, which have been known for over 40 years, were by far the most wellcited and persistent finding. Other reported effects included observations of: glucocorticoid and androgen metabolism, glycolysis and Krebs cycle, gluconeogenesis, fatty acid metabolism, fatty acid oxidation and pantothenic acid, amino acid oxidation, tryptophan metabolism and effects on microbiome metabolites. The discussion section draws observations from the very disparate studies together and links them to the general aspects of human metabolism.
\end{abstract}

Keywords (6-8): Human, metabolomics, microbiome, physical activity, purine metabolism, training effect.

\section{INTRODUCTION}

Physical activity and exercise are potent lifestyle interventions [1] for the reduction of several psycho-somatic risks, including cardiovascular disease (CVD) [2], diabetes [2] and mild-to-moderate depression [1]. According to the most recent reports of the World Health Organisation [3] CVDs were the leading cause of mortality (17.5 million deaths) followed by diabetes ( 1.5 million deaths). In the UK this translates to more than 200,000 (37\% of total) deaths due to CVD [4]. In fact, the five leading global risks for mortality are, to a certain degree, modifiable risk factors including, high blood pressure (13\%), tobacco use (9\%), high blood glucose $(6 \%)$, physical inactivity $(6 \%)$ and overweight/obesity (5\%) [5]. These risks can be modified by effects elicited by fruit and vegetable consumption (diet modification), moderate alcohol use and physical activity. It has been estimated that the indirect cost of physical inactivity in England is $£ 8.2$ billion per annum [6], in developed countries this is $1.5 \%-3 \%$ of total direct healthcare costs [7], and finally Scotland is one of the least physically active countries in Europe [8]. Regular exercise, a relatively inexpensive treatment [1], can modify but more importantly improve overall quality of life and life expectancy. However, questions remain with regard to adequate duration, frequency and intensity, as well as what type of exercise is optimal for individuals of different cohorts (i.e. sedentary vs. already active), and what the molecular mechanisms underlying health

\footnotetext{
*Address correspondence to this author at the Strathclyde Institute of Pharmacy and Biomedical Sciences, The John Arbuthnott Building, 161 Cathedral Street, Glasgow G4 0RE; Tel: (+44) (0) 1415482 651; E mail: d.g.watson@strath.ac.uk
}

improvement through exercise are. The answer may depend on various factors such as gender, age, life- style and body mass index (BMI). However, directly investigating the effect of exercise on the human metabolome, via metabolomicsbased techniques, can provide new insight into novel phenotypic responses- giving rise to personalised training regimes that are reflective of the initial metabolic status of each individual.

Surprisingly, in spite of the importance of exercise in maintaining health, there are only around 64 papers in the literature that have investigated the effects of exercise on the metabolome during the past 15 years (1999-2014). Within the metabolomics discipline, systematic and high throughput procedures aim to identify and quantify the full set of metabolites within a defined system at a specified time-point in response to internal as well as external stimuli. The term metabolomics itself was only formally coined just over a decade ago, although the fundamental instrumental techniques go back to the 1960s [9]. Increases in the power of mass spectrometry (MS) and nuclear magnetic resonance (NMR) techniques coupled to advances in data extraction and manipulation techniques have led to a rapid growth in publications on metabolomics. The most recurring theme with regard to the metabolic effects of exercise in the literature is related to purine metabolism and more specifically to hypoxanthine a purine derivative and intermediate in the adenosine metabolism. Adenosine metabolism is important in exercise because of the crucial role of adenosine triphosphate (ATP) in muscle function. The earliest observed increase in "nonuric acid purine" in urine samples postexercise was made in the early 1900s by Burian (as referenced in Nasrallah and Al- Khalidi [10]). Most investiga- 
tions into these adenine nucleotides, as potent vasodilators and contributors in energy regulation, trace back as far as the techniques developed in the 1960s that have formed the basis of metabolomics. Starting with human studies, Forrester and Lind (1969) investigated the ATP content from venous effluent of the forearm muscle in response to exercise (i.e. $10 \%$ and $20 \%$ maximal voluntary contractions), illustrating an increase in ATP post-exercise compared to resting values [11]. Frog and other animal heart (intact and perfused) studies were used, in the 1930 s, in order to demonstrate an improvement in heart beat after injections of nucleic acid derivatives [11, 12]. From around 1999 onwards the principal metabolite of interest in a variety of biological fluids for monitoring the effect of exercise is hypoxanthine. Thus a broader consideration of the metabolites affected by exercise is due.

The current review aims to identify the research studies which have utilised a metabolomics/metabolite profiling methodologies to monitor the effects of exercise in predominantly healthy subjects in the period 1999-2014. Based on these criteria 64 papers were selected through the following search engines, Google Scholar (http://scholar.google.co.uk/) and Pubmed (http://www.ncbi.nlm.nih.gov/pubmed). Fig. (1) illustrates the main features of the included studies ranging from metabolomics technology, subject group demographics, gender groups as well as sample type tested. A summary table (Table 1) has been created for each study identified highlighting the main metabolomics technology used, subject demographics, exercise regime and sampling timepoints. Table 2 contains a summary of the sample type tested along with main metabolites that were analysed via the metabolomics technology accompanied by relative response to exercise where appropriate. The majority of the studies are focused on particular pathways and most of the untargeted metabolomics studies on exercise that have been published within the last four years are in line with the wider availabil- ity of high resolution MS and more advanced NMR techniques. There is, however, a large variation in the depth of the studies as judged by their success in generating intriguing new hypotheses where there is a sense of the metabolome operating in a complex and dynamic fashion in response to a variety of exercise regimes. The discussion in this review will be focused more on the in depth studies, and the ones not included have been placed as supplementary material in Tables $\mathbf{1}$ and $\mathbf{2}$. We have also included a selection of metabolomics-based studies where exercise was used as a health intervention in the last three years (2011-2014).

\section{STUDIES FOCUSED ON PURINE METABOLISM}

Observations on the effects of exercise on purine metabolism go back over 40 years. Normally during exercise the energy expenditure is closely related to ATP utilisation. However, during high-intensity exercise the demand for ATP outstrips supply and this causes degradation of total adenine nucleotides $[\mathrm{TAN}=\mathrm{ATP}+\mathrm{ADP}$ (adenosine diphosphate) + AMP] via deamination of AMP (adenosine 5'monophosphate) to form IMP (inosine monophosphate) which produces ammonia as a side product [13]. The IMP can then be lost from the muscle cells in the form of hypoxanthine which accumulates in plasma. Yet, hypoxanthine can be salvaged by hypoxanthine-guanine phosphoribosyl transferase (HGPRT). Several papers have observed that the hypoxanthine salvage process tends to be more efficient in trained individuals and that, along with other purine metabolites, its concentrations in blood can provide an indication of the effectiveness of a training regimen- that can also be illustrated by an improvement in the level of ATP re-synthesis [14]. Following exhaustive exercise muscle urate levels were also shown to increase [15]. Interestingly, the non-enzymatic oxidation of urate results in the formation of allantoin which makes it possible to assess to what extent it is acting as an antioxidant.
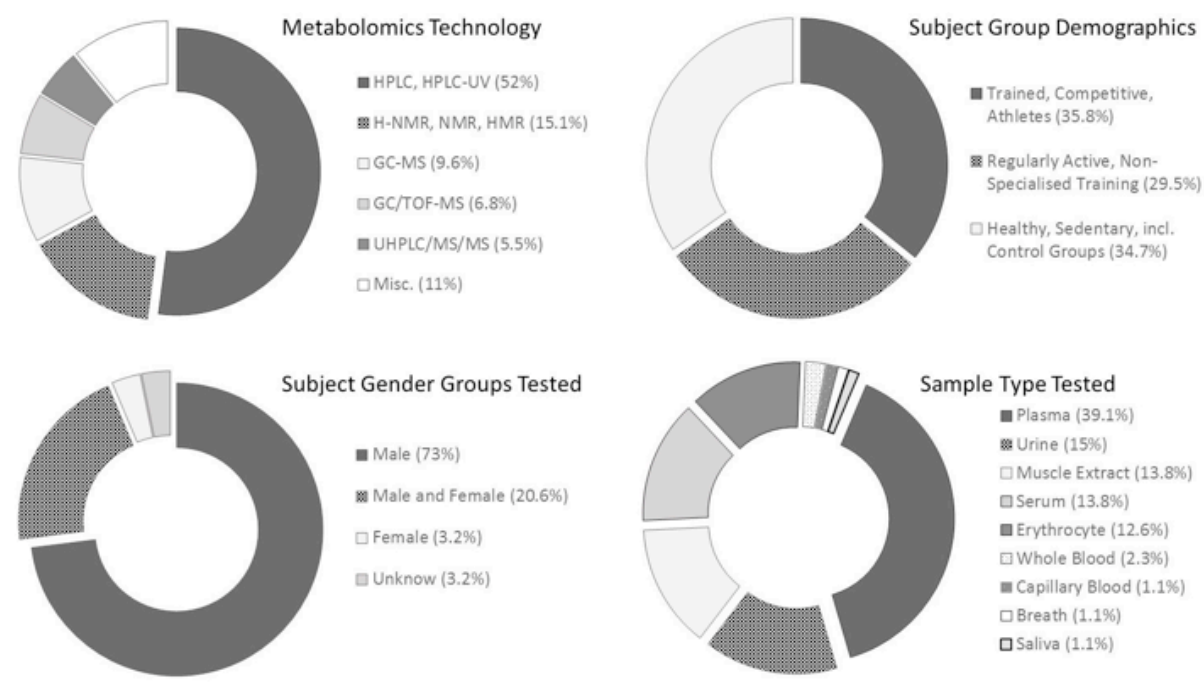

Fig. (1). Main features of the research studies selected for the current review excluding patient cohort studies. The most prevalent metabolomics technology utilised is high performance liquid chromatography (HPLC), via plasma of healthy trained/athlete and active male groups.

Abbreviations: HPLC-UV: high performance liquid chromatography- ultraviolet detection; ${ }^{1} \mathrm{H}-\mathrm{NMR}$ : proton nuclear magnetic resonance; GC-MS: gas chromatography mass spectrometry; GC/TOF-MS: gas chromatography time-of-flight mass spectrometry; UHPLC/MS/MS: ultra-high performance liquid chromatography tandem mass spectrometry. Miscellaneous includes: LC-APCI-MS: liquid chromatography- atmospheric pressure chemical ionisation- mass spectrometry; (FIA)-MS/MS: flow injection analysis tandem mass spectrometry; PTR MS: proton transfer reaction mass spectrometry; (FIESI-ICR-FT)/MS: Flow injection electrospray ionization ion cyclotron resonance Fourier transform mass spectrometry; CE: capillary electrophoresis; HPLC-ESI-MS-MS: high performance liquid chromatography- electrospray ionisation- tandem mass spectrometry; UPLC-qTOF-MS: ultraperformance liquid chromatography coupled with electrospray time-of-flight mass spectrometry. 
Table 1. Detailed Study Features of Metabolomics-based Articles* since 1999 with Main Study Aim to Investigate Effect of Exercise on Different Human Bio-fluid Metabolomes.

\begin{tabular}{|c|c|c|c|c|c|}
\hline Study & Instrument & Exercise Test & $\begin{array}{l}\text { Subject Characteristics } \\
\text { (N, Gender, Age, Test Groups) }\end{array}$ & Sampling Time-Points & $\begin{array}{l}\text { Ref. } \\
\text { No. }\end{array}$ \\
\hline Sahlin, et al. & $\overline{\text { HPLC }}$ & $\begin{array}{l}\text { Session 1- Incremental } \dot{V} \mathrm{O}_{2 \max } \text { test, } \\
\text { cycle ergometer } \\
\text { Session 2- } \\
\text { 1. 5min warm-up @ } 50 \% \dot{V} \mathrm{O}_{2 \max } \\
2.75 \% \dot{V} \mathrm{O}_{2 \max }(60 \mathrm{rpm}) \text { until v.e. }\end{array}$ & $\begin{array}{l}\text { 10, Male, } 23-32 \text { yrs } \\
\text { One Group- active }\end{array}$ & $\begin{array}{l}\text { Plasma- rest (pre), during- } \\
\text { exercise (every } 20 \mathrm{~min} \text { and at } \\
\text { exhaustion), 5min post-exercise } \\
\text { Muscle (lateral aspect } \text { of the } \\
\text { quadriceps) extracts- pre- } \\
\text { exercise and post-exercise }\end{array}$ & [13] \\
\hline Hellsten, et al. & HPLC & $\begin{array}{l}\text { Session 1- } \dot{V} \mathrm{O}_{2 \max } \text { test, cycle ergome- } \\
\text { ter } \\
\text { 1.10min warm-up @ } 100 \mathrm{~W}, 80 \mathrm{rpm} \\
\text { 2.3min rest } \\
\text { 3.6min @ } 150-170 \mathrm{~W} \\
\text { 4.5min rest } \\
\text { 5.6min @ } 225-275 \mathrm{~W} \\
6.5 \mathrm{~min} \text { rest } \\
\text { 7. cycle to exhaustion@ @ 275W w/ 25W } \\
\text { increase ever } 45 \mathrm{~s} \\
\text { Session 2- Cycle } 90 \% \\
\text { exhaustion } \\
\text { Session 3- Work load adjusted to elicit } \\
\text { exhaustion after 15-20min } \\
\text { Session 4- 2x cycle to exhaustion @ } \\
263 \pm 13 \mathrm{~W} \text {, bouts separated by } 1 \mathrm{hr}\end{array}$ & $\begin{array}{l}\text { 8, Male, } 21 \text { - 25yrs } \\
\text { One Group- active }\end{array}$ & $\begin{array}{l}\text { Plasma- rest (pre), } 1,5,10,15, \\
\text { and } 17.5 \text { min during-exercise, } \\
\text { and immediately before exhaus- } \\
\text { tion, as well as at } 1,2,4,9,20 \text {, } \\
30,45 \text {, and } 60 \text { min post-exercise } \\
\text { Muscle (vastus lateralis) ex- } \\
\text { tracts- rest (pre) and at exhaus- } \\
\text { tion } \\
\text { [samples taken at session } 4 \text { for } \\
\text { each bout] }\end{array}$ & {$[14]$} \\
\hline Stathis, et al. & $\begin{array}{l}\text { HPLC } \\
\text { [Merck Hibar Li- } \\
\text { chrosphere } 100 \mathrm{CH}-18 / 2 \\
250 \mathrm{~mm} \times 4 \mathrm{~mm} \text { column] }\end{array}$ & $\begin{array}{l}\text { Session 1- } \mathrm{O}_{2 \text { peak }} \text { test, cycle ergome- } \\
\text { ter } \\
\text { Session 2- 1wk sprint training } \\
\text { [twice each day } 1 \text { set of } 15 \times 10 \mathrm{~s} \text { 'all- } \\
\text { out' sprints w/ } 50 \text { s break between } \\
\text { sprint, two sets separated by minimum } \\
6 \mathrm{hr} \text { rest] } \\
\text { Performance Tests (PT): Pre-sessions } 2 \\
\text { a 30s 'all-out' sprint was performed } \\
\text { followed by another post } 1 \text { wk training }\end{array}$ & $\begin{array}{l}\text { 7, Male, } 23.1 \pm 1.8 y r s \\
\text { One Group- active, non-specialised } \\
\text { training }\end{array}$ & $\begin{array}{l}\text { Plasma- rest (pre), } 0,10,15,20 \text {, } \\
30,60 \text { and } 120 \mathrm{~min} \text { post-PT } \\
\text { Urine- } 12 \mathrm{hrs} \text { pre and } 2,6 \text {, and } \\
16 \mathrm{hrs} \text { post-PT } \\
\text { Muscle extract- rest, immedi- } \\
\text { ately post-exercise and } 10 \mathrm{~min} \\
\text { into recovery }\end{array}$ & {$[15]$} \\
\hline Zielińksi, et al. & $\begin{array}{l}\text { HPLC } \\
\text { [Hypersil ODS } 100 \mathrm{~mm} \times \\
4.6 \mathrm{~mm} \times 5 \mu \mathrm{m} \text { column } \\
\text { and a Hypersil ODS } \\
20 \mathrm{~mm} \times 4 \mathrm{~mm} \times 5 \mu \mathrm{m} \text { pre- } \\
\text { column] }\end{array}$ & $\begin{array}{l}4 \text { testing sessions across } 1 \mathrm{yr} \text { training } \\
\text { cycle } \\
\text { [preparatory phase: } 1 \text {; competition } \\
\text { phase: } 1 \text {; transition phase: } 1 \text {; end of } 1 \mathrm{yr} \\
\text { training cycle and during next yr pre- } \\
\text { paratory phase: } 1 \text { ] } \\
\text { Session } 1,2,3 \text { and } 4 \text { - Incremental } \\
\quad \mathrm{O}_{2 \max } \text { test, treadmill } \\
{\left[10 \mathrm{~km} / \mathrm{hr}^{-1} \text { start, then } 2 \mathrm{~km} / \mathrm{hr}^{-1} \text { increase }\right.} \\
\text { every } 3 \mathrm{~min} \text { until v.e.] }\end{array}$ & $\begin{array}{l}\mathbf{9} \text {, Male, } 22.9 \pm 0.6 y r s \\
\text { One Group- long distance runners }\end{array}$ & $\begin{array}{l}\text { Rest, immediately pre-exercise } \\
\text { and } 5 \mathrm{~min} \text { post-exercise }\end{array}$ & {$[16]$} \\
\hline Zielińksi, et al. & $\begin{array}{l}\text { HPLC } \\
\text { [Hypersil ODS } 100 \mathrm{~mm} \times \\
4.6 \mathrm{~mm} \times 5 \mu \mathrm{m} \text { column } \\
\text { and a Hypersil ODS } \\
20 \mathrm{~mm} \times 4 \mathrm{~mm} \times 5 \mu \mathrm{m} \text { pre- } \\
\text { column] }\end{array}$ & $\begin{array}{l}4 \text { testing sessions across } 1 \text { yr training } \\
\text { cycle } \\
\text { [preparatory phase: } 1 \text { pre and post } 1^{\text {st }} \\
\text { specific subphase; competition phase: } 1 \\
\text { pre; and transition phase: } 1 \text { post] } \\
\text { Session 1- Incremental } \mathrm{O}_{2 \max } \text { test, } \\
\text { treadmill } \\
{\left[10 \mathrm{~km} / \mathrm{hr}^{-1} \text { start, then } 2 \mathrm{~km} / \mathrm{hr}^{-1} \text { increase }\right.} \\
\text { every } 3 \mathrm{~min}^{-} \text {until v.e., measuring VT } \\
\text { and HR] } \\
\text { For sessions } 2,3 \text { and } 4 \text { training loads } \\
\text { adjusted based on } 5 \text { energetic zones }\end{array}$ & $\begin{array}{l}\text { Group 1- 11, Male, } 22.3 \pm 0.7 y r s \\
\text { Middle-distance runners } \\
\text { Group 2- 11, Male, } 24.1 \pm 1.9 y r s \\
\text { No prior/current competitive sport } \\
\text { experience, control group }\end{array}$ & $\begin{array}{l}\text { Rest, immediately pre-exercise } \\
\text { and } 5 \mathrm{~min} \text { post-exercise }\end{array}$ & [17] \\
\hline Zielińksi, et al. & $\begin{array}{l}\text { HPLC } \\
\text { [Hypersil ODS } 100 \mathrm{~mm} \times \\
4.6 \mathrm{~mm} \times 5 \mu \mathrm{m} \text { column } \\
\text { and a Hypersil ODS } \\
20 \mathrm{~mm} \times 4 \mathrm{~mm} \times 5 \mu \mathrm{m} \text { pre- } \\
\text { column] }\end{array}$ & $\begin{array}{l}4 \text { testing sessions across } 1 \mathrm{yr} \text { training } \\
\text { cycle } \\
\text { [preparatory phase: } 2 \text {; competition } \\
\text { phase: } 1 \text {; and transition phase: } 1 \text { ] } \\
\text { Session } 1,2,3 \text { and } 4 \text { - Incremental } \\
\quad \mathrm{O}_{2 \max } \text { test, treadmill } \\
\text { [10km/hr }{ }^{-1} \text { start, then } 2 \mathrm{~km} / \mathrm{hr}^{-1} \text { increase } \\
\text { every } 3 \mathrm{~min} \text { until v.e.] }\end{array}$ & $\begin{array}{l}\text { Group 1- 10, Male, 20 - 29yrs } \\
\text { Sprinters } \\
\text { Group 2- 10, Male, } 21 \text { - 28yrs } \\
\text { Triathletes } \\
\text { Highly trained competitive athletes }\end{array}$ & $\begin{array}{l}\text { Rest, immediately pre-exercise } \\
\text { and } 5 \mathrm{~min} \text { post-exercise }\end{array}$ & [18] \\
\hline
\end{tabular}


(Table 1) contd....

\begin{tabular}{|c|c|c|c|c|c|}
\hline Study & Instrument & Exercise Test & $\begin{array}{l}\text { Subject Characteristics } \\
\text { (N, Gender, Age, Test Groups) }\end{array}$ & Sampling Time-Points & $\begin{array}{l}\text { Ref. } \\
\text { No. }\end{array}$ \\
\hline Zielińksi, et al. & $\begin{array}{l}\text { HPLC } \\
\text { [Hypersil ODS } 100 \mathrm{~mm} \times \\
4.6 \mathrm{~mm} \times 5 \mu \mathrm{m} \text { column } \\
\text { and a Hypersil ODS } \\
20 \mathrm{~mm} \times 4 \mathrm{~mm} \times 5 \mu \mathrm{m} \text { pre- } \\
\text { column] }\end{array}$ & $\begin{array}{l}\text { 1yr training cycle incl. preparatory, } \\
\text { competitive and transition phases } \\
\text { Session 1- Incremental } \dot{V} \mathrm{O}_{2 \max } \text { test } \\
\text { precompetitive phase, treadmill } \\
\text { [start @ } 10 \mathrm{~km} / \mathrm{hr}^{-1} \mathrm{w} / 2 \mathrm{~km} / \mathrm{hr}^{-1} \text { increase } \\
\text { every } 3 \mathrm{~min} \text { until v.e.] }\end{array}$ & $\begin{array}{l}\text { 71, Male, Healthy and Highly } \\
\text { Trained Athletes } \\
\text { Group } 1-28,19-28 \mathrm{yrs} \text {, triathletes } \\
\text { Group 2- 12, 20-26yrs, long- } \\
\text { distance runners } \\
\text { Group 3- } 13,20-26 \mathrm{yrs} \text {, middle- } \\
\text { distance runners } \\
\text { Group 4- } 18,19-29 \mathrm{yrs} \text { sprinters }\end{array}$ & $\begin{array}{l}\text { Pre-exercise and 5min post- } \\
\text { exercise }\end{array}$ & 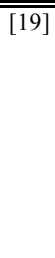 \\
\hline Zielińksi, et al. & $\begin{array}{l}\text { HPLC } \\
\text { [Hypersil ODS } 100 \mathrm{~mm} \times \\
4.6 \mathrm{~mm} \times 5 \mu \mathrm{m} \text { column } \\
\text { and a Hypersil ODS } \\
20 \mathrm{~mm} \times 4 \mathrm{~mm} \times 5 \mu \mathrm{m} \text { pre- } \\
\text { column] }\end{array}$ & $\begin{array}{l}3 \text { testing sessions across 1yr training } \\
\text { cycle } \\
\text { [preparatory phase: } 1 \text {; competition } \\
\text { phase: } 1 \text {; and transition phase: } 1 \text { ] } \\
\text { Session 1- Incremental } \dot{V} \mathrm{O}_{2 \max } \text { test, } \\
\text { treadmill } \\
\text { [start @ } 10 \mathrm{~km} / \mathrm{hr}^{-1} \mathrm{w} / 2 \mathrm{~km} / \mathrm{hr}^{-1} \text { increase } \\
\text { every } 3 \mathrm{~min} \text { until v.e.] } \\
\text { Training Loads: } \\
\text { Group } 1-7 \text { times a week, total weekly } \\
\text { training time of } 8-10 \mathrm{hrs} \text {, number of } \\
\text { training session in } 1 \text {-year cycle was } 315 \\
\text { Group } 2-5 \text { times a week, total weekly } \\
\text { training time of } 5 \mathrm{hrs,} \mathrm{number} \mathrm{of} \mathrm{train-} \\
\text { ing session in } 1 \text {-year cycle was } 221 \\
\text { Group } 3-3 \text { times a week, total weekly } \\
\text { training time of } 3 \mathrm{hrs,} \mathrm{number} \mathrm{of} \mathrm{train-} \\
\text { ing session in } 1 \text {-year cycle was } 123\end{array}$ & $\begin{array}{l}\text { 30, Male, Middle-aged Runners } \\
\text { Group } 1-11,46 \pm 3.8 \mathrm{yrs} \text {, elite master } \\
\text { runners } \\
\text { Group } 2-9,45.1 \pm 4.7 \mathrm{yrs} \text {, amateur } \\
\text { runners } \\
\text { Group 3- } 10,45.9 \pm 6.1 \mathrm{yrs} \text {, recrea- } \\
\text { tional runners }\end{array}$ & $\begin{array}{l}\text { Pre-exercise and } 5 \mathrm{~min} \text { post- } \\
\text { exercise }\end{array}$ & {$[20]$} \\
\hline Dudzinska, et al. & HPLC & $\begin{array}{l}\text { Session 1- Continuous effort test } \mathrm{w} / \\
\text { progressively increasing intensity } \\
\text { 1. } 5 \mathrm{~min} @ 25 \mathrm{~W} \text {, warm-up } \\
\text { 2. Start } \rightarrow 70 \mathrm{~W} \text {, cadence 70rpm } \\
\text { 3. } 20 \mathrm{~W} \text { increase every } 3 \text { min until max } \\
\text { effort reached }\end{array}$ & $\begin{array}{l}\text { 22, Male, 21.9 } \pm 2.33 \text { yrs } \\
\text { One Group- healthy }\end{array}$ & $\begin{array}{l}\text { Immediately pre- and post- } \\
\text { exercise, and } 30 \mathrm{~min} \text { post- exer- } \\
\text { cise }\end{array}$ & {$[21]$} \\
\hline Bianchi, et al. & HPLC & $\begin{array}{l}\text { Group 1- All out 4min aerobic/ an- } \\
\text { aerobic exercise } \\
\text { Group 2- Soccer match } \\
\text { Group 3- Marathon }\end{array}$ & $\begin{array}{l}\text { 52 athletes } \\
\text { Group 1- 8, cycle pursuers, 20-25yrs } \\
\text { Group 2- 19, soccer players, 17-20yrs } \\
\text { Group 3- 25, marathon runners, 22- } \\
\text { 34yrs }\end{array}$ & $\begin{array}{l}\text { Pre-exercise and } 5 \mathrm{~min} \text { of cessa- } \\
\text { tion }\end{array}$ & {$[23]$} \\
\hline Gerber, et al. & $\begin{array}{l}\text { HPLC } \\
{[\text { Gemini C18,5 } \mu(150 \times} \\
4 \mathrm{~mm}) \text { column] }\end{array}$ & 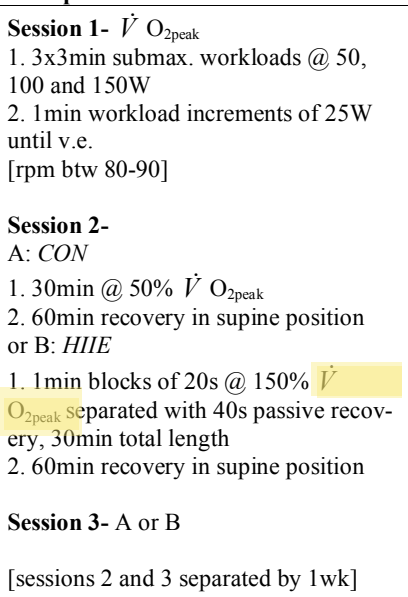 & $\begin{array}{l}\text { 8, Male, 18- 35yrs } \\
\text { One Group- healthy untrained }\end{array}$ & $\begin{array}{l}\text { Plasma at rest, during exercise } \\
\text { at } 1,3,5,10,15 \text {, and } 30 \mathrm{~min} \text { and } \\
\text { at } 5,10,20,30 \text {, and } 60 \mathrm{~min} \text { post- } \\
\text { exercise } \\
\text { Urine collected overnight } \\
\text { before trial and following } 60 \mathrm{~min} \\
\text { post-exercise. }\end{array}$ & {$[24]$} \\
\hline Stathis, et al. & HPLC & $\begin{array}{l}\text { Session 1- } \dot{V} \mathrm{O}_{2 \text { peak }} \text { test, cycle ergome- } \\
\text { ter } \\
\text { [3min at } 3 \text { submx work rates followed } \\
\text { by }>\text { work rate every min until v.e.] } \\
\text { Session } 2-5 \text { days of ingestion of } \\
\text { placebo (calcium carbonate) OR } \\
\text { allopurinol (300mg tablet) followed } \\
\text { by } 8 \times 10 \text { s 'all-out' sprints w/ } 50 \text { s break } \\
\text { between sprints. } \\
\text { Session } 3-5 \text { days of ingestion of } \\
\text { placebo OR allopurinol (reversed) } \\
\text { followed by } \\
8 \times 10 \mathrm{~s} \text { 'all-out' sprints w/ } 50 \mathrm{~s} \text { break } \\
\text { between sprints. }\end{array}$ & $\begin{array}{l}\text { 7, Male, } 24.9 \pm 3 y r s \\
\text { Active but non-specifically trained } \\
{\left[\dot{V} \mathrm{O}_{2 \text { peak }} \text { of } 48.1 \pm 6.9 \mathrm{~mL} \cdot \mathrm{kg}^{-1} \cdot \mathrm{min}^{-1}\right]}\end{array}$ & $\begin{array}{l}\text { Plasma- rest (pre), } 0,10,15,20 \text {, } \\
30,60 \text { and } 120 \mathrm{~min} \text { post- final } \\
\text { sprint } \\
\text { Urine- } 12 \mathrm{hrs} \text { pre and } 2,6 \text {, and } \\
16 \mathrm{hrs} \text { post-PT }\end{array}$ & {$[25]$} \\
\hline
\end{tabular}


(Table 1). contd....

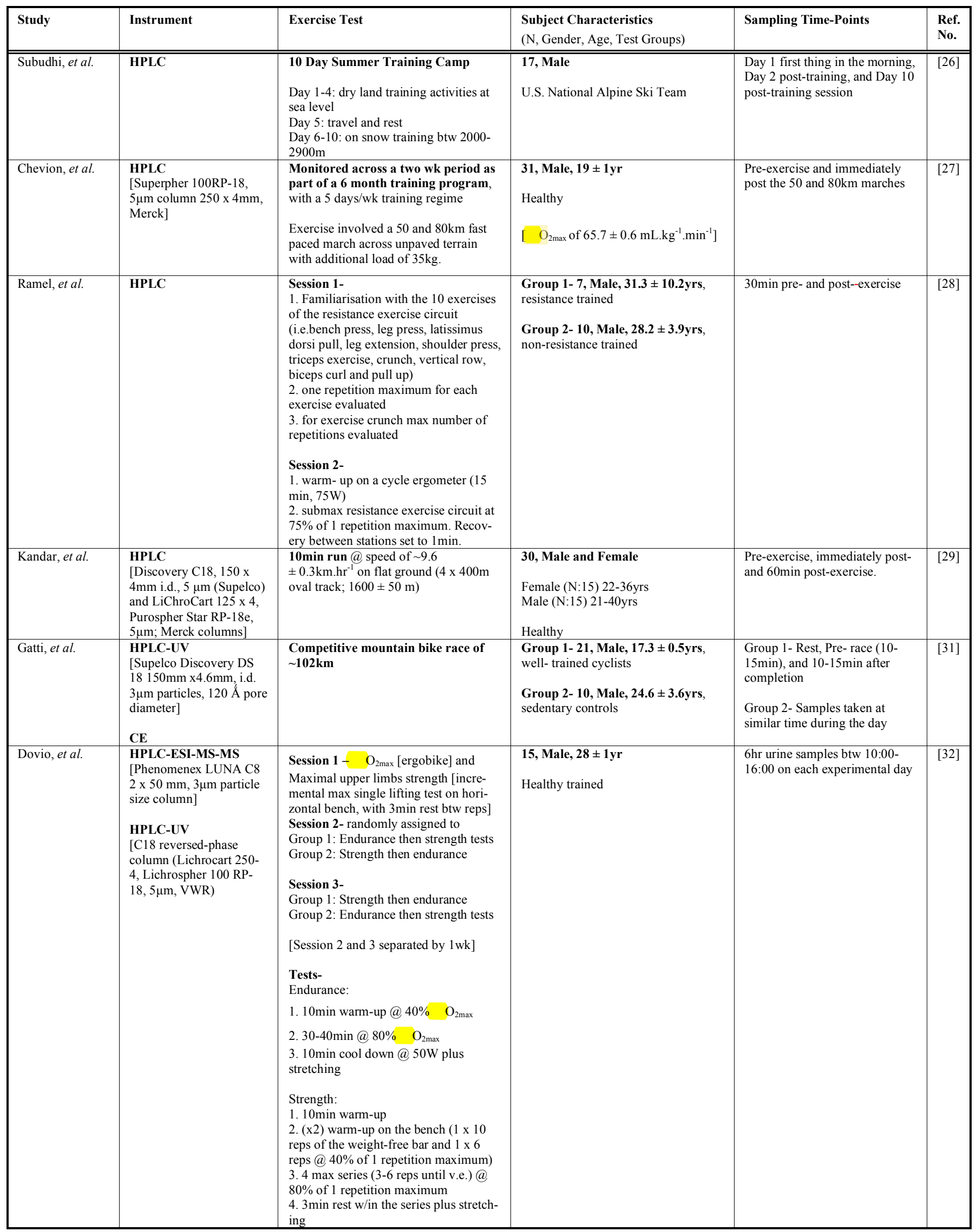


(Table 1) contd....

\begin{tabular}{|c|c|c|c|c|c|}
\hline Study & Instrument & Exercise Test & $\begin{array}{l}\text { Subject Characteristics } \\
\text { (N, Gender, Age, Test Groups) }\end{array}$ & Sampling Time-Points & $\begin{array}{l}\text { Ref. } \\
\text { No. }\end{array}$ \\
\hline Giraldo, et al. &  & $\begin{array}{l}\text { Session 1- } \mathrm{O}_{2 \max } \\
\text { [ergometer, no specific protocol de- } \\
\text { scribed] } \\
\text { Then assigned to: } \\
\text { Group 1 (N:15)- } 45 \mathrm{~min} @ 55 \% \quad \mathrm{O}_{2 \max } \\
\text { Group 2 (N:15)- } 1 \mathrm{hr} @ 70 \% \quad \mathrm{O}_{2 \max }\end{array}$ & $\begin{array}{l}\text { 30, Female, 20-24yrs } \\
\text { Healthy sedentary untrained with not } \\
\text { involved in exercise program in the } \\
\text { previous } 24 \text { months pre testing } \\
\text { [Testing occurred during follicular } \\
\text { phase of menstrual cycle] }\end{array}$ & $\begin{array}{l}\text { Pre-exercise, immediately post- } \\
\text { exercise and } 24 \mathrm{hr} \text { post }\end{array}$ & 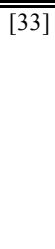 \\
\hline Degoutte, et al. & $\begin{array}{l}\text { HPLC } \\
{[\mathrm{C} 18 \text { column }]}\end{array}$ & $\begin{array}{l}\text { Session 1- } \\
\text { 1. } 20 \mathrm{~min} \text { warm-up } \\
\text { 2. } 10 \mathrm{~min} \text { rest } \\
\text { 3. } 5 \text { min judo match }\end{array}$ & $\begin{array}{l}\text { 16, Male, } 18.4 \pm 1.6 y r s \\
\text { National judoists } \\
{\left[\quad \mathrm{O}_{2 \max } \text { of } 55.7 \pm 0.5 \mathrm{~mL} \cdot \mathrm{kg}^{-1} \cdot \mathrm{min}^{-1}\right]}\end{array}$ & $\begin{array}{l}\text { Rest, } 3 \mathrm{~min}, 1 \text { and } 24 \mathrm{hr} \text { post- } \\
\text { match }\end{array}$ & {$[36]$} \\
\hline Gangemi, et al. & $\begin{array}{l}\text { HPLC } \\
\text { [Waters Symmetry C18, } \\
3 \mu \mathrm{m}, 2.1 \times 150 \mathrm{~mm} \text { col- } \\
\text { umn] }\end{array}$ & $\begin{array}{l}\text { Session 1- } \\
\text { Exercise test on cycle ergometer } \\
\text { 1. initial load set to } 25 \mathrm{~W} \text { for } 2 \mathrm{~min} \\
\text { 2. }>25 \mathrm{~W} \text { every } 2 \mathrm{~min} \text { until subject } \\
\text { theoretical heart rate max reached }\end{array}$ & $\begin{array}{l}\text { 9, Male and Female, } 29.3 \pm 3.5 y r s \\
\text { healthy }\end{array}$ & $\begin{array}{l}\text { Pre-exercise, } 3 \mathrm{~min} \text { post- exer- } \\
\text { cise, } 6 \text { and } 24 \mathrm{hr} \text { into recovery }\end{array}$ & {$[37]$} \\
\hline Sparling, et al. & $\begin{array}{l}\text { HPLC/MS } \\
\text { [Hewlett Pckard octade- } \\
\text { cyl silica Hypersil colum } \\
(100 \times 4.6 \mathrm{~mm} \text { i.d., } 5 \mu \mathrm{m}) \\
\text { reversed-phase] }\end{array}$ & $\begin{array}{l}\text { Group } 1 \text { and 2- } \\
\text { Either on treadmill or cycle ergometer } \\
\text { 1. } 5 \mathrm{~min} \text { warm-up } \\
\text { 2. > work rate to elicit heart rate of } 70 \text { - } \\
80 \% \text { of } \max \\
\text { 3. sustain for } 45 \mathrm{~min} \\
\text { Group 3- } \\
\text { Seated for } 50 \mathrm{~min}\end{array}$ & $\begin{array}{l}\mathbf{2 4} \text {, Males, } 23.7 \pm 9.4 y r s \\
\text { Exercising for } 6 \text { months pre-trial } \\
\text { ( } \geq 30 \mathrm{~min} \text { running/cycling, } \geq 4 / \mathrm{wk} \text { ) } \\
\text { Assigned to following groups depend- } \\
\text { ing on pre-training regime: } \\
\text { Group } 1(\mathrm{~N}: 8) \text { - Running } \\
\text { Group } 2(\mathrm{~N}: 8)-\text { Cycling } \\
\text { Group } 3(\mathrm{~N}: 8) \text { - Control }\end{array}$ & Pre- and post--exercise sessions & {$[38]$} \\
\hline Dudzinska, et al. & HPLC & $\begin{array}{l}\text { Continuous effort and progressive } \\
\text { cycle test } \\
\text { 1. } 5 \text { min warm-up @ } 25 \mathrm{~W} \\
\text { 2. Increase to } 70 \mathrm{~W} \text { w/ } 70 \mathrm{rpm} \\
\text { 3. }>20 \mathrm{~W} \text { every } 3 \mathrm{~min} \text { until v.e. or until } \\
\text { unable to maintain } 70 \mathrm{rpm} \text {. }\end{array}$ & $\begin{array}{l}\text { 44, Male, } 18.5 \pm 2.92 \mathrm{yrs} \\
\text { One Group- healthy } \\
{\left[\mathrm{O}_{2 \max }: 50.2 \pm 6.26 \mathrm{~mL} \cdot \mathrm{kg}^{-1} \cdot \mathrm{min}^{-1}\right]}\end{array}$ & $\begin{array}{l}\text { Pre-exercise, immediately post- } \\
\text { and } 30 \mathrm{~min} \text { post-exercise. }\end{array}$ & [39] \\
\hline Lewis, et al. & $\mathbf{L C} / \mathbf{M S} / \mathbf{M S}^{\mathrm{a}}$ & $\begin{array}{l}\text { Group 1- Cardiopulmonary exercise } \\
\text { testing [bicycle ergometer, 10-25W/min } \\
\text { with > ramp cycle] } \\
\text { Group 2- Exercise treadmill testing } \\
\text { [Bruce protocol] } \\
\text { Group 3- Boston Marathon }\end{array}$ & $\begin{array}{l}\text { Group 1- } 8 \text {, Male and Female, } 48 \pm \\
14 \text { yrs } \\
\text { Group 2- } \\
\text { (a) } 40, \text { Male and Female, } 58 \pm 13 \text { yrs } \\
\text { (b) } 25 \text { (validation group), Male and } \\
\text { Female, } 59 \pm 12 \text { yrs } \\
\text { Group 3- } 25 \text {, Male and Female, } 42 \pm \\
\text { 9yrs } \\
\text { Amateur runners }\end{array}$ & $\begin{array}{l}\text { Group 1- Plasma from the } \\
\text { superior vena cava and pulmo- } \\
\text { nary artery at baseline, peak } \\
\text { exercise and } 60 \mathrm{~min} \text { post- } \\
\text { exercise } \\
\text { Group 2- Plasma at baseline, } \\
\text { peak exercise and } 60 \mathrm{~min} \text { post- } \\
\text { exercise } \\
\text { Group 3- Plasma at pre-exercise } \\
\text { and post-exercise }\end{array}$ & {$[40]$} \\
\hline Pohjanen, et al. & GC/TOF-MS & $\begin{array}{l}\text { Session 1- } \mathrm{O}_{2 \text { peak }} \text { test, cycle ergome- } \\
\text { ter } \\
\text { Session 2-90min cycle } \\
\text { [each } 90 \mathrm{~min} \text { cycle consists of } 9 \times 10 \mathrm{~min} \\
\text { equal sets] } \\
\text { Sets of } 10 \mathrm{~min} \text { each: } \\
1.2 \mathrm{~min} @ 40 \% \\
2.6 \mathrm{~min} @ 60 \% \\
3.2 \mathrm{~min} @ 85 \% \quad \mathrm{O}_{2 \text { peak }} \\
\text { [Session } 2 \text { completed in total } 4 \text { times, } \\
1 \text { wk apart permitted per session] }\end{array}$ & $\begin{array}{l}\mathbf{2 4} \text {, Male, } 25.7 \pm 2.7 \mathrm{yrs} \\
\text { One Group- healthy, regularly trained } \\
\mathrm{O}_{2 \text { peak }}: 59.1 \pm 7.3 \mathrm{~mL} \cdot \mathrm{kg}^{-1} \cdot \mathrm{min}^{-1}\end{array}$ & $\begin{array}{l}\text { Pre-exercise and immediately } \\
\text { post-exercise }\end{array}$ & [41] \\
\hline Yan, et al. & GC/TOF-MS & $\begin{array}{l}\text { 2wk Training Program } \\
{[11 \text { sessions/wk, } 30 \mathrm{hrs} \text { in total of tech- }} \\
\text { nical and aerobic exercise] }\end{array}$ & $\begin{array}{l}\text { Male } \\
\text { Group 1- junior trained rowers, } 20.2 \pm \\
1.1 \mathrm{yrs} \\
\text { Group 2- senior trained rowers, } 23.0 \pm \\
2.7 \mathrm{yrs} \\
\text { Group 3- sex and age matched con- } \\
\text { trols, healthy }\end{array}$ & $\begin{array}{l}\text { Pre-training, at } 1 \mathrm{wk} \text { and post } \\
2 \mathrm{wk} \text { training }\end{array}$ & {$[42]$} \\
\hline $\begin{array}{l}\text { Pechlivanis, et } \\
\text { al. }\end{array}$ & $\begin{array}{l}{ }^{1} \mathbf{H}-\mathbf{N M R} \\
{[5 \mathrm{~mm} \text { inverse probe }]}\end{array}$ & $\begin{array}{l}\text { Group A Session- } 3 \text { sets of } 2 \times 80 \mathrm{~m} \\
\text { max runs, the two runs separated by } 10 \mathrm{~s} \\
\text { rest } \\
\text { Group B Session- } 3 \text { sets of } 2 \times 80 \mathrm{~m} \\
\text { max runs, the two runs separated by } 1 \mathrm{~m} \\
\text { rest } \\
{[20 \mathrm{~min} \text { rest between sets for both }} \\
\text { groups] }\end{array}$ & $\begin{array}{l}\text { 12, Male, Moderately Trained } \\
\text { Group A- } 21 \pm 2 \text { yrs } \\
\text { Group B- } 20 \pm 1 \text { yrs }\end{array}$ & $\begin{array}{l}\text { Pre-exercise and } 35 \mathrm{~min} \text { post- } \\
\text { exercise }\end{array}$ & {$[43]$} \\
\hline
\end{tabular}


(Table 1) contd....

\begin{tabular}{|c|c|c|c|c|c|}
\hline Study & Instrument & Exercise Test & $\begin{array}{l}\text { Subject Characteristics } \\
\text { (N, Gender, Age, Test Groups) }\end{array}$ & Sampling Time-Points & $\begin{array}{l}\text { Ref. } \\
\text { No. }\end{array}$ \\
\hline $\begin{array}{l}\text { Pechlivanis, et } \\
\text { al. }\end{array}$ & 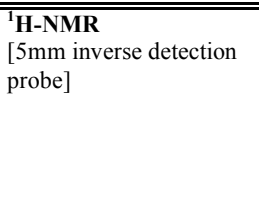 & $\begin{array}{l}\text { 8wks training programme with } 3 \\
\text { exercise sessions per week. } \\
1 . \text { First } 4 \mathrm{wks}=\text { each session incl. } 2 \text { sets } \\
\text { of } 80 \mathrm{~min} \text { max runs } \\
\text { 2. Second } 4 \mathrm{wks}=\text { each sessions incl. } 3 \\
\text { sets of } 80 \mathrm{~min} \text { max runs }\end{array}$ & $\begin{array}{l}\text { 14, Male, Healthy and Moderately } \\
\text { Trained } \\
\text { Group A- } 21 \pm 2 \text { yrs, each run per } \\
\text { session separated by } 10 \text { s rest } \\
\text { Group B- } 20 \pm 2 \text { yrs, each run per } \\
\text { session separated by } 1 \mathrm{~min} \text { rest }\end{array}$ & $\begin{array}{l}\begin{array}{l}\text { Pre-exercise and } 30 \mathrm{~min} \text { post- } \\
\text { exercise } \\
\text { [Before and after } 8 \mathrm{wks} \text { of train- } \\
\text { ing] }\end{array}\end{array}$ & 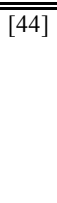 \\
\hline Mukherjee, et al. & $\begin{array}{l}{ }^{1} \mathbf{H}-\mathbf{N M R} \\
{[5 \mathrm{~mm} \text { broadband probe] }}\end{array}$ & $\begin{array}{l}\text { Session 1- } \dot{V} \mathrm{O}_{2 \text { peak }} \\
\text { Session 2- } \\
1.6 \mathrm{~min} \text { cycling @ } 25 \mathrm{~W} \text { with cadence } \\
80 \mathrm{rpm} \text {, } \\
2.45 \mathrm{~min} \text { cycling @ } 60 \% \mathrm{WR}_{\text {peak }} \text {, } \\
3.90 \% \mathrm{WR}_{\text {peak }} \text { until v.e. }\end{array}$ & $\begin{array}{l}\mathbf{1 7} \text {, Male, 50- 60yrs } \\
\text { Group 1- } 9 \text { master athletes } \\
\text { Group 2- } 8 \text { healthy untrained }\end{array}$ & $\begin{array}{l}\text { Pre-exercise, immediately post- } \\
\text { exercise and } 24 \mathrm{hr} \text { post-exercise }\end{array}$ & [45] \\
\hline Santone, et al. & $\begin{array}{l}{ }^{1} \text { H-NMR } \\
{[5 \mathrm{~mm} \text { triple resonance }} \\
\text { probe] }\end{array}$ & $\begin{array}{l}\text { Session 1- } \\
\text { 1. Warm-up (running) } \\
\text { 2. YO-YO Test, } 2 \times 20 \mathrm{~m} \text { shuttle runs } \\
\text { with } 10 \mathrm{~s} \text { recovery between bouts, } \\
\text { starting speed } 10 \mathrm{~km}^{-1} \mathrm{hr}^{-1}\end{array}$ & $\begin{array}{l}\text { 14, Male, } 23 \pm 3 \text { yrs } \\
\text { One Group- healthy elite soccer } \\
\text { players }\end{array}$ & Pre-exercise and post-exercise & [46] \\
\hline Kujala, et al. & ${ }^{1} \mathrm{H}-\mathrm{NMR}$ & $\begin{array}{l}\text { Longitudinal physical activity meas- } \\
\text { ured with the Metabolic Equivalent } \\
\text { (MET) index. }\end{array}$ & $\begin{array}{l}\text { PART 1: } \\
\text { Finnish Twin Cohort Study } \\
\text { 32, male and female same-sex twins, } \\
50-74 \text { yrs } \\
\text { Group 1- } 16 \text { healthy active } \\
\text { Group 2- } 16 \text { healthy inactive } \\
\text { PART 2: } \\
\text { Pieksämaki Cohort Study } \\
\text { 230, male and female, 41-62yrs } \\
\text { Group 1- 115 healthy active } \\
\text { Group 2- } 115 \text { healthy inactive } \\
\text { PART 3: } \\
\text { Young Finns Study } \\
\text { 600, male and female, 30-45yrs } \\
\text { Group 1- 300 healthy active } \\
\text { Group 2- } 300 \text { healthy inactive } \\
\text { PART 4: } \\
\text { Northern Finland Birth Cohort } \\
\text { 1966 } \\
\text { 1244, male and female, 31yrs } \\
\text { Group 1- } 622 \text { healthy active } \\
\text { Group 2- } 622 \text { healthy inactive }\end{array}$ & $\begin{array}{l}\text { PART 1: Single sample post } \\
\text { 32yrs of follow-up (2007) } \\
\text { PART 2: Single sample post } \\
\text { 5yrs of follow up (2003 and } \\
\text { 2004) } \\
\text { PART 3: Single sample post } \\
\text { 5yrs of follow up (2007) } \\
\text { PART 4: Single sample post } \\
\text { 5yrs of follow up (1980 and } \\
\text { 1997-1998) }\end{array}$ & {$[48]$} \\
\hline Netzer, et al. & Targeted MS/MS $^{b}$ & $\begin{array}{l}\text { Session 1- Incremental bicycle ergome- } \\
\text { ter test }^{\mathrm{c}} \\
\text { [Increments increase by } 25 \mathrm{~W} \text { per step } \\
\text { until maximum capacity reached] }\end{array}$ & $\begin{array}{l}\text { 30, Male and Female, } 38.33 \pm \\
7.16 y r s \\
\text { One Group- active and physically fit }\end{array}$ & $\begin{array}{l}\text { Pre-exercise, at each step until } \\
\text { (incl.) max performance }\end{array}$ & [49] \\
\hline Lehmann, et al. & $\begin{array}{l}\text { UPLC-qTOF-MS } \\
\text { HPLC }\end{array}$ & $\begin{array}{l}\text { Session 1- } \\
\text { 1. Running velocity @ individual } \\
\text { anaerobic threshold, determined with an } \\
\text { incremental exercise test, treadmill } \\
{\left[6 \mathrm{~km} / \mathrm{h}^{-1},>2 \mathrm{~km} / \mathrm{h}^{-1} \text { every } 3 \mathrm{~min}\right]} \\
\text { 2. } \quad \mathrm{O}_{2 \max }, \text { ramp test } \\
{\left[8 \mathrm{~km} / \mathrm{h}^{-1},>1 \mathrm{~km} / \mathrm{h}^{-1} \text { every } 0.5 \text { min until }\right.} \\
\text { v.e.] } \\
\text { Session 2- } \\
\text { Group 1: 60min continuous run of } \\
\text { moderate intensity } \\
93 \% \text { of the VIAT }\left(\sim 75 \% \text { of } \quad \mathrm{O}_{2 \max }\right) \\
\text { Group } 2: 120 \text { min continuous run } \\
70 \% \text { of the VIAT }\left(\sim 55 \% \quad \mathrm{O}_{2 \max }\right)\end{array}$ & $\begin{array}{l}\text { Group 1- 13, Male, } 32.6 \pm \mathbf{6 . 1 y r s} \\
\mathrm{O}_{2 \max }: 56.5 \pm 1.4 \mathrm{~mL} \cdot \mathrm{kg}^{-1} \cdot \mathrm{min}^{-1} \\
\text { Group 2- 8, Male, } \mathbf{3 0 . 9} \pm \mathbf{5 . 8 y r s} \\
\mathrm{O}_{2 \max }: 63.0 \pm 2.0 \mathrm{~mL} \cdot \mathrm{kg}^{-1} \cdot \mathrm{min}^{-1} \\
\text { [both groups consisted of healthy, } \\
\text { lean subjects] }\end{array}$ & $\begin{array}{l}\text { Pre-exercise and immediately } \\
\text { post, } 3 \mathrm{hr} \text { post and } 24 \mathrm{hr} \text { post }\end{array}$ & [50] \\
\hline
\end{tabular}


(Table 1) contd....

\begin{tabular}{|c|c|c|c|c|c|}
\hline Study & Instrument & Exercise Test & $\begin{array}{l}\text { Subject Characteristics } \\
\text { (N, Gender, Age, Test Groups) }\end{array}$ & Sampling Time-Points & $\begin{array}{l}\text { Ref. } \\
\text { No. }\end{array}$ \\
\hline$\overline{\text { Enea, et al. }}$ & $\begin{array}{l}{ }^{1} \mathbf{H}-\mathbf{N M R} \\
{[5 \mathrm{~mm} \text { broadband inverse }} \\
\text { probe }]\end{array}$ & $\begin{array}{l}\text { Session 1- } \\
\text { (a) Progressive incremental } \dot{V} \mathrm{O}_{2 \max } \\
\text { test, cycle ergometer } \\
\text { 1. } 4 \text { min warm-up @ } 75 \mathrm{~W} \\
\text { 2. Power output increased by } 25 \mathrm{~W} \\
\text { every min until v.e. } \\
\text { (b) Four } 6 \text { s sprints against increasing } \\
\text { braking force }\left(1^{\text {st }} \text { set } 2 \mathrm{~kg} \text { then }>\text { by } 1 \mathrm{~kg}\right. \\
\text { per sprint), } 5 \text { min recovery after each } \\
\text { sprint } \\
\text { Session 2- } \\
\text { 1. } 30 \mathrm{~s} \text { sprint on cycle ergometer } @ \text { max } \\
\text { speed, individually determined resis- } \\
\text { tance } \\
\text { 2. } 90 \mathrm{~min} \text { rest in recumbent position } \\
\text { Session 3- } \\
1.4 \mathrm{~min} \text { warm-up, } 75 \mathrm{~W} \\
\text { 2. } 70 \% \dot{V} \text { O } \mathrm{O}_{2 \max } \text { until v.e. } \\
\text { 3. } 5 \mathrm{~min} \text { active recovery } \\
\text { 4. } 90 \mathrm{~min} \text { rest in recumbent position }\end{array}$ & $\begin{array}{l}\begin{array}{l}\mathbf{2 2}, \text { Female } \\
\text { [on oral contraceptive pills for } 6 \\
\text { months] }\end{array} \\
\text { Group 1- } 9, \mathbf{2 2 . 1} \pm \mathbf{0 . 6 y r s , ~ u n t r a i n e d ~} \\
\text { recreational exercisers } \\
\text { Group 2- 12, 21.8 } \pm \mathbf{1 . 0 y r s ,} \\
\text { trained (6: judoists; 6: cy- } \\
\text { clists/triathletes) }\end{array}$ & $\begin{array}{l}\text { Pre-exercise and 30min post } \\
\text { each exercise session }\end{array}$ & 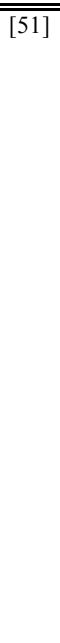 \\
\hline Neal, et al. & ${ }^{1} \mathrm{H}-\mathrm{NMR}$ & $\begin{array}{l}\text { 29wk Training Program } \\
\text { 1. } 2 \text { wks general testing } \\
\text { [2x } 40 \mathrm{~km} \text { time trial, peak power output } \\
\text { test, cycle ergometer] } \\
\text { 2. 4wks detraining } \\
\text { 3. 1wk general testing } \\
\text { 4. } 6 \text { wks POL or THR, } 3 \text { days/wk } \\
\text { 5. 1wk general testing } \\
\text { 6. 4wk detraining } \\
\text { 7. 1wk general testing } \\
\text { 8. 6wks POL or THR, } 3 \text { days } / w k \\
\text { 9. 1wk general testing } \\
\text { Polarised training model (POL) training } \\
\text { intensity: } 80 \% \text { low intensity, } 0 \% \text { mod- } \\
\text { erate intensity, } 20 \% \text { high-intensity } \\
\text { Threshold training distribution } \\
\text { (THR) training intensity: } 57 \% \text { low } \\
\text { intensity, } 40 \% \text { moderate intensity, } 0 \% \\
\text { high-intensity }\end{array}$ & $\begin{array}{l}\mathbf{1 2} \text {, Male, } 37 \pm \mathbf{6 y r s} \\
\text { One Group Cross Over Design- } \\
\text { Trained road cyclists }\end{array}$ & [during general testing wk] & {$[52]$} \\
\hline Peake, et al. & $\begin{array}{l}\text { HPLC } \\
\text { GC-MS } \\
\text { [ZB1701(Zebron) capil- } \\
\text { lary column }(30 \mathrm{~m} \text { x } 250 \\
\mu \mathrm{m}(\mathrm{ID}) \times 0.15 \mu \mathrm{m} \text { film } \\
\text { thickness] }\end{array}$ & $\begin{array}{l}\text { Session 1, 2- } \mathrm{O}_{2 \max } \\
\text { Graded exercise test on cycle ergometer } \\
\text { until v.e. } \\
1.5-10 \mathrm{~min} @ \text { self-selected intensity } \\
2 . \text { Test start at } 100 \mathrm{~W} \text { and power }>\text { by } \\
15 \mathrm{~W} \text { every } 30 \mathrm{~s} \text { until v.e. } \\
\text { [cadence kept at } \geq 60 \text { r.p.m] } \\
\text { Session 3- High-intensity interval } \\
\text { training (HIIT) familiarisation } \\
10 \mathrm{x} \text { min intervals } @ \text { power output } \\
\text { corresponding to } \sim 80 \% \quad \mathrm{O}_{2 \text { max }} \text { within } \\
\text { a } 2 \text { min recovery @ } 50 \mathrm{~W} \text { btw intervals. } \\
\text { [Familiarisation conducted } 48 \mathrm{hr} \text { post } 2^{\text {nd }} \\
\quad \mathrm{O}_{2 \text { max }} \text { and used to determine duration } \\
\text { required to complete the same work rate } \\
\text { on a cycle with continuous power } \\
\text { output } \sim 65 \% \quad \mathrm{O}_{2 \text { max }} \text { which is for the } \\
\text { moderate-intensity continuous exercise } \\
\text { (MOD) } \\
\text { Session } 4 \text { - randomly assigned to HIIT } \\
\text { or MOD then reversed } \\
\text { [at least } 1 \text { wk post familiarisation and } 7 \\
\text { day break between MOD or HIIT] }\end{array}$ & $\begin{array}{l}\text { 10, Male, } 33.2 \pm 6.7 \mathrm{yrs} \\
\text { Well trained cyclists and triathletes } \\
{\left[\mathrm{O}_{2 \max }: 4.8 \pm 0.3 \mathrm{~L} / \mathrm{min}\right]}\end{array}$ & $\begin{array}{l}\text { Pre-exercise, immediately post- } \\
\text { exercise, } 1 \text { and } 2 \text { hrs post- } \\
\text { exercise }\end{array}$ & {$[53]$} \\
\hline
\end{tabular}


(Table 1) contd....

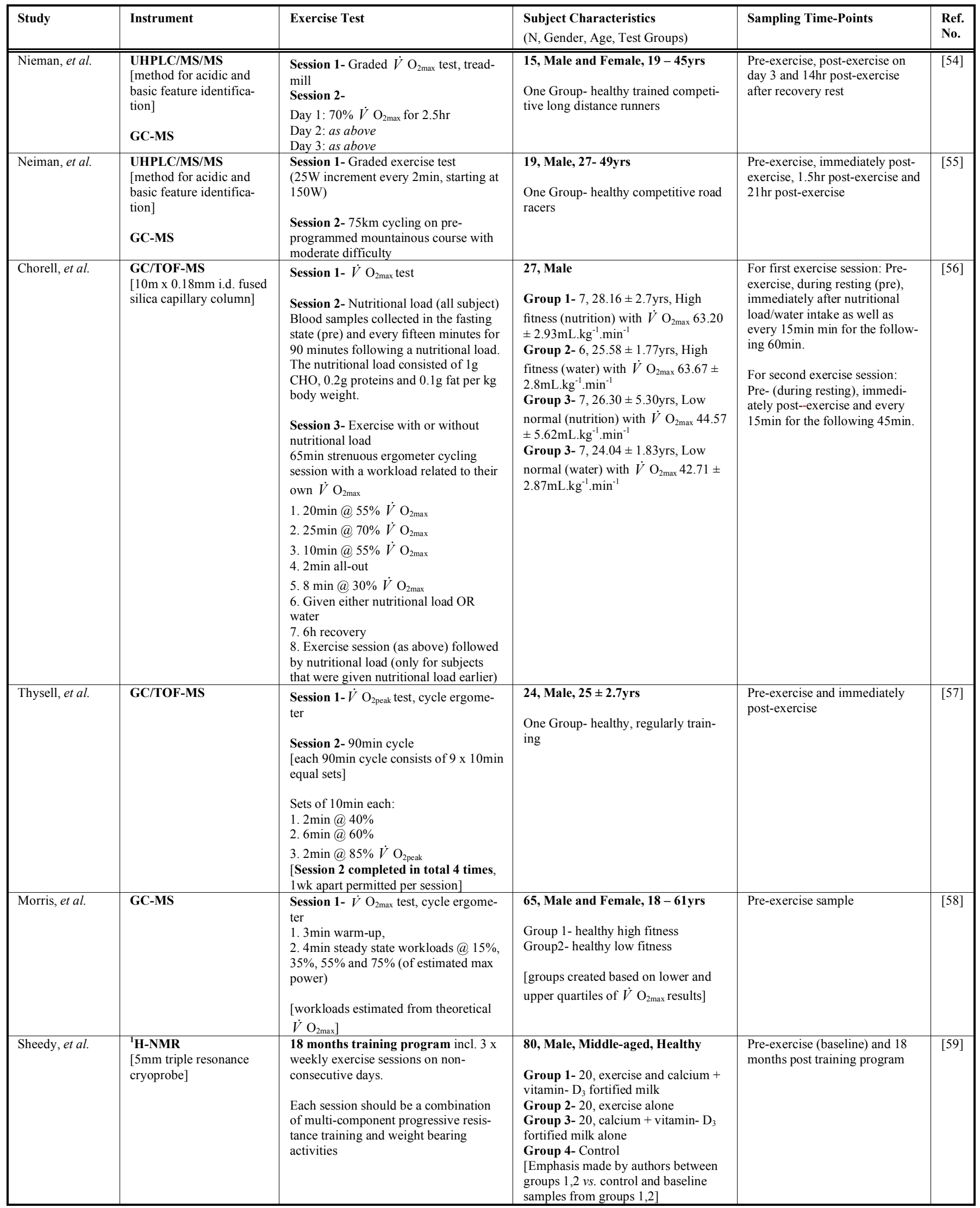


(Table 1) contd....

\begin{tabular}{|c|c|c|c|c|c|}
\hline Study & Instrument & Exercise Test & $\begin{array}{l}\text { Subject Characteristics } \\
\text { (N, Gender, Age, Test Groups) }\end{array}$ & Sampling Time-Points & $\begin{array}{l}\text { Ref. } \\
\text { No. }\end{array}$ \\
\hline$\overline{\text { Nieman, et al. }}$ & $\begin{array}{l}\text { UHPLC/MS/MS } \\
\text { [method for acidic and } \\
\text { basic feature identifica- } \\
\text { tion] } \\
\text { GC-MS } \\
\text { [C18 Sep Pak column] }\end{array}$ & $\begin{array}{l}\text { Session 1- Graded } \dot{V} \mathrm{O}_{2 \max } \text { test, tread- } \\
\text { mill } \\
\text { Session 2- } 2 \text { wks of supplementation } \\
\text { with either polyphenol soy protein } \\
\text { complex or placebo accompanied by } \\
\text { normal training. } \\
\text { Session 3- } 3 \text { days running bout exercise } \\
(2.5 \mathrm{~h} / \text { day on treadmill @ } ~ 70 \% \\
\left.\dot{V} \mathrm{O}_{2 \max }\right) \text { accompanied by supplements. }\end{array}$ & $\begin{array}{l}\text { 31, Male and Female, Competitive } \\
\text { half and full marathon road racers } \\
\text { Group 1- } 16 \text {, Male and Female, } 33.7 \\
\pm 6.8 \mathrm{yrs}, \text { intervention group } \\
\text { Group 2- } 15 \text {, Male and Female, } 35.2 \\
\pm 8.7 \mathrm{yrs}, \text { placebo group }\end{array}$ & $\begin{array}{l}\text { Pre-day } 1 \text { exercise, post- day } 3 \\
\text { exercise and } 14 \mathrm{hrs} \text { post-exercise } \\
\text { at day } 4\end{array}$ & [60] \\
\hline Nieman, et al. & $\begin{array}{l}\text { GC-MS } \\
\text { [C18 Sep Pak column] }\end{array}$ & $\begin{array}{l}\text { Session 1- Graded exercise test } \\
(25 \mathrm{~W}>\text { every } 2 \mathrm{~min} \text {, starting at } 150 \mathrm{~W}) \\
\text { Session 2- } 75 \mathrm{~km} \text { time trial ( } 1 \mathrm{wk} \text { post } \\
\text { session } 1) \\
\text { Randomly assigned to bananas OR } 6 \% \\
\text { CHO } \\
\text { Session 3- } 75 \mathrm{~km} \text { time trial ( } 3 \mathrm{wk} \text { post } \\
\text { session } 2) \\
\text { Reversed supplement conditions. } \\
\text { [Prior to session } 2 \text { and } 3 \text { subjects in- } \\
\text { gested } 0.4 \mathrm{~g} / \mathrm{kg} \text { carbohydrate from } \\
\text { bananas OR from a standard } 6 \% \mathrm{CHO} \text {. } \\
\text { During exercise subjects ingested } 0.2 \\
\text { g/kg body weight every } 15 \mathrm{~min} \text { of } \\
\text { bananas or CHO] }\end{array}$ & $\begin{array}{l}\text { 14, Male, 18-45yrs } \\
\text { Competitive road cyclists }\end{array}$ & $\begin{array}{l}\text { Pre-exercise and supplementa- } \\
\text { tion, immediately post- } 75 \mathrm{~km} \\
\text { time trial, and } 1 \mathrm{hr} \text { post-exercise }\end{array}$ & {$[61]$} \\
\hline Chorell, et al. & $\begin{array}{l}\text { GC/TOF-MS } \\
{[10 \mathrm{~m} \times 0.18 \mathrm{~mm} \text { i.d. fused }} \\
\text { silica capillary column }]\end{array}$ & $\begin{array}{l}\text { Session- } \mathrm{O}_{2 \text { peak }} \\
\text { Session 2- } 90 \mathrm{~min} \text { of ergometer cycling } \\
\text { Each 10min set repeated } 9 \text { times with- } \\
\text { out rest: } 2 \mathrm{~min} @ 40 \%, 6 \text { min } @ 60 \% \\
\text { and } 2 \mathrm{~min} @ 85 \% \quad \mathrm{O}_{2 \text { peak }} \\
1 \text { of } 4 \text { beverages immediately post } \\
\text { completion of the } 90 \text { min cycling, each } \\
\text { subject completed all } 4 \text { tests in a ran- } \\
\text { domized order, ingesting } 1 \text { beverage per } \\
\text { test. } 100 \mathrm{~mL} \text { of water was ingested as } \\
\text { well as at }+15,+30 \text {, and }+60 \text { min post- } \\
\text { exercise } \\
\text { Beverage 1- } 5 \mathrm{dL} \text { water } \\
\text { Beverage } 2-\text { low low CHO }(1 \mathrm{~g} \mathrm{CHO} \\
\text { per kg body weight in water }(16 \% \mathrm{w} / \mathrm{v})) \\
\text { Beverage } 3-\text { high CHO }(1.5 \mathrm{~g} \mathrm{CHO} \mathrm{per} \\
\text { kg body weight in water }(24 \% \mathrm{w} / \mathrm{v})) \\
\text { Beverage } 4-\text { low CHO-protein }(1 \mathrm{~g} \mathrm{CHO} \\
\text { per kg ody weight in water }(16 \% \mathrm{w} / \mathrm{v})+ \\
0.5 \mathrm{~g} \text { protein per kg body weight })\end{array}$ & $\begin{array}{l}\text { 24, Male, } 25.7 \pm \mathbf{2 . 7 y r s} \\
{\left[\mathrm{O}_{2 \text { peak }} 59.1 \pm 7.3 \mathrm{~mL} \cdot \mathrm{kg}^{-1} \cdot \mathrm{min}^{-1}\right]}\end{array}$ & $\begin{array}{l}\text { Pre-exercise, immediately post } \\
\text { and at } 15,30,60 \text { and } 90 \mathrm{~min} \\
\text { post-exercise }\end{array}$ & [62] \\
\hline
\end{tabular}

Abbreviations: $\dot{V} \mathrm{O}_{2 \max }$ : maximal oxygen consumption; CON: constant steady state exercise; HIE: high intermittent exercise; W: Watts; WR ${ }_{\text {peak: }}$ peak work rate; v.e.: volitional exhaustion; rpm: revolutions per minute; VT: Ventilator threshold; HR: heart rate; TT: time trial.

${ }^{*}$ Articles have been presented in order of appearance.

atriple quadrupole mass spectrometer operated in electrospray ionization mode using a turbo ion spray LC-MS interface.

${ }^{b}$ No details given by authors regarding specific metabolomics-based technology and method.

${ }^{\mathrm{c}}$ No details given by authors regarding specific exercise testing protocol. 
Table 2. Metabolite Details as Presented from the Exercise Metabolomics Literature and Identified Through a Metabolomics-based Technology.

\begin{tabular}{|c|c|c|c|}
\hline Study & $\begin{array}{l}\text { Sample Tested } \\
\text { (Plasma:P; Erythrocytes:E; Urine:U; Serum: S; Muscle } \\
\text { Extract:ME; Saliva:Sa, Breath:B; Whole Blood:WB; Capil- } \\
\text { lary Blood:CB) }\end{array}$ & $\begin{array}{l}\text { Metabolite Pathways } \\
\text { ( } \downarrow \text { : reduced post- exercise, } \uparrow: \text { increased post-exercise) }\end{array}$ & $\begin{array}{l}\text { Ref. } \\
\text { No. }\end{array}$ \\
\hline Sahlin, et al. & $\mathrm{P}$ and $\mathrm{ME}$ & $\begin{array}{l}\text { Hypoxanthine, } \uparrow(\mathbf{P}) \\
\text { Xanthine, } \uparrow(\mathbf{P}) \\
\text { Urate, } \uparrow(\mathbf{P}) \\
\text { IMP } \\
\text { ATP } \\
\text { AMP }\end{array}$ & [13] \\
\hline $\begin{array}{l}\text { Hellsten, et } \\
\text { al. }\end{array}$ & $\mathrm{P}$ and ME (Vastus Lateralis) & $\begin{array}{l}\text { IMP, } \uparrow \text { (ME) } \\
\text { Hypoxanthine, } \uparrow \text { (P) } \\
\text { Urate, } \uparrow \text { (ME) } \\
\text { Allantoin, } \uparrow \text { (ME) }\end{array}$ & [14] \\
\hline $\begin{array}{l}\text { Zielińksi, et } \\
\text { al. }\end{array}$ & $P$ and $E$ & $\begin{array}{l}\text { Hypoxanthine (P) } \\
\text { Xanthine }(\mathrm{P}) \\
\text { Uric Acid }(\mathrm{P}) \\
\text { HGPRT (E) } \\
\text { (Significant changes observed for each metabolite across the } 1 \text { year training } \\
\text { cycle) }\end{array}$ & [16] \\
\hline $\begin{array}{l}\text { Zielińksi, et } \\
\text { al. }\end{array}$ & $P$ and $E$ & $\begin{array}{l}\text { Hypoxanthine (P) } \\
\text { Xanthine }(\mathrm{P}) \\
\text { Uric acid }(\mathrm{P}) \\
\text { HGPRT (E) } \\
\text { (Higher anaerobic exercise training decreases post-exercise hypoxanthine and } \\
\text { uric acid with an increase in HGPT- this relationship is reversed when athlete } \\
\text { reaches transition phase) }\end{array}$ & [17] \\
\hline $\begin{array}{l}\text { Zielińksi, et } \\
\text { al. }\end{array}$ & $P$ and $E$ & $\begin{array}{l}\text { Hypoxanthine (P), good predictor of fitness } \\
\text { Xanthine (P) } \\
\text { Uric acid (P) } \\
\text { HGPRT (E) }\end{array}$ & [19] \\
\hline $\begin{array}{l}\text { Zielińksi, et } \\
\text { al. }\end{array}$ & $P$ and $E$ & $\begin{array}{l}\text { Hypoxanthine (P), Group1 continuous } \downarrow \text { across three tests, Group2 } \downarrow \text { in } 1^{\text {st }} \\
\text { and } 2^{\text {nd }} \text { tests, Group } 3 \text { no change } \\
\text { HGPRT (E), Group1 continuous } \uparrow \text { across three tests, Group2 } \uparrow \text { in } 1^{\text {st }} \text { and } 2^{\text {nd }} \\
\text { tests, Group } 3 \text { no change }\end{array}$ & {$[20]$} \\
\hline $\begin{array}{l}\text { Dudzinska, } \\
\text { et al. }\end{array}$ & WB and $\mathrm{E}$ & $\begin{array}{l}\text { ADP, } \downarrow \text { (E) post 30min recovery } \\
\text { AMP, } \downarrow \text { (E) post 30min recovery } \\
\text { IMP, } \uparrow \text { (E) immediately post and } \downarrow \text { (E) post 30min recovery } \\
\text { Inosine, } \downarrow \text { (WB) post 30min recovery } \\
\text { Hypoxanthine, } \uparrow \text { (WB) immediately post, } \uparrow \text { (WB) post 30min recovery }\end{array}$ & [21] \\
\hline
\end{tabular}


(Table 2) contd....

\begin{tabular}{|c|c|c|c|}
\hline Study & $\begin{array}{l}\text { Sample Tested } \\
\text { (Plasma:P; Erythrocytes:E; Urine:U; Serum: S; Muscle } \\
\text { Extract:ME; Saliva:Sa, Breath:B; Whole Blood:WB; Capil- } \\
\text { lary Blood:CB) }\end{array}$ & $\begin{array}{l}\text { Metabolite Pathways } \\
\text { ( } \downarrow \text { : reduced post--exercise, } \uparrow: \text { increased post-exercise) }\end{array}$ & $\begin{array}{l}\text { Ref. } \\
\text { No. }\end{array}$ \\
\hline $\begin{array}{l}\text { Bianchi, et } \\
\text { al. }\end{array}$ & $P$ & Hypoxanthine, $\uparrow$ & {$[23]$} \\
\hline Gerber, et al. & $\mathrm{P}$ and $\mathrm{U}$ & $\begin{array}{l}\text { Hypoxanthine, } \uparrow(\mathbf{P}) \\
\text { Uric acid, } \uparrow(\mathbf{P \& U})\end{array}$ & {$[24]$} \\
\hline Stathis, et al. & $P$ and $U$ & $\begin{array}{l}\text { Inosine, } \uparrow(\mathbf{P}) \\
\text { Hypoxanthine, } \uparrow(\mathbf{P}) \text { greater in allopurinol vs. placebo same in (U) } \\
\text { Xanthine, } \uparrow(\mathbf{U}) \text { greater in allopurinol vs. placebo }\end{array}$ & {$[25]$} \\
\hline $\begin{array}{l}\text { Subudhi, et } \\
\text { al. }\end{array}$ & $P$ & $\alpha$ - and $\gamma$-tocopherol, $\downarrow$ over training camp & {$[26]$} \\
\hline $\begin{array}{l}\text { Chevion, et } \\
\text { al. }\end{array}$ & $\mathrm{P}$ & $\begin{array}{l}\text { Uric Acid, } \uparrow \\
\text { Ascorbic acid } \\
\text { Dehydroascorbate }\end{array}$ & {$[27]$} \\
\hline Ramel, et al. & $P$ & $\begin{array}{l}\alpha \text { - and } \gamma \text {-tocopherol } \\
\beta \text {-carotene } \\
\text { Lycopene } \\
\text { Ascorbic Acid } \\
\text { Malondialdehyde, } \uparrow \text { in Group1, } 2 \\
\text { Linoleic acid }\end{array}$ & {$[28]$} \\
\hline $\begin{array}{l}\text { Kandar, et } \\
\text { al. }\end{array}$ & $\mathrm{P}$ and $\mathrm{E}$ & $\begin{array}{l}\text { Allantoin, } \uparrow(\mathbf{P \& E}) \\
\text { Uric acid, } \uparrow(\mathbf{P \& E}) \\
\text { Malondialdehyde, } \uparrow(\mathbf{E})\end{array}$ & {$[29]$} \\
\hline Gatti, et al. & $\mathrm{U}$ & $\begin{array}{l}\text { Cortisol, } \uparrow \\
\text { Cortisone, } \uparrow \\
\text { Creatinine }\end{array}$ & {$[31]$} \\
\hline Dovio, et al. & $\mathrm{U}$ & $\begin{array}{l}\text { Total Cortisol } \\
\text { Cortisone } \\
\text { Tetrahydroderivatives } \\
\text { Unconjugated Cortisol and Cortisone } \\
\text { [tetra- hydrocortisol (THF) }+ \text { alloTHF]/tetrahydrocortisone ratio, } \uparrow\end{array}$ & {$[32]$} \\
\hline $\begin{array}{l}\text { Giraldo, et } \\
\text { al. }\end{array}$ & $\mathrm{P}$ & $\begin{array}{l}\text { Norepinephrine, } \uparrow \text { post-exercise vs. pre in Group1, } 2 \text { and } \downarrow \text { post } 24 \text { hours } \\
\text { recovery in Group1, } 2 \\
\text { Epinephrine, } \uparrow \text { post-exercise vs. pre in Group2 and } \downarrow \text { post } 24 \text { hours recovery } \\
\text { in Group2 }\end{array}$ & {$[33]$} \\
\hline $\begin{array}{l}\text { Degoutte, et } \\
\text { al. }\end{array}$ & $\mathrm{P}$ & $\begin{array}{l}\text { Xanthine, } \uparrow \\
\text { Hypoxanthine, } \uparrow\end{array}$ & {$[36]$} \\
\hline $\begin{array}{l}\text { Gangemi, et } \\
\text { al. }\end{array}$ & $\mathrm{U}$ & $\begin{array}{l}\text { Lipoxin } \\
\text { Tetraene }\end{array}$ & {$[37]$} \\
\hline $\begin{array}{l}\text { Sparling, et } \\
\text { al. }\end{array}$ & $\mathrm{P}$ & Anandamide, $\uparrow$ in runners and cyclists & {$[38]$} \\
\hline $\begin{array}{l}\text { Dudzinska, } \\
\text { et al. }\end{array}$ & $\mathrm{P}$ and $\mathrm{E}$ & Uridine $\uparrow \uparrow$ & {$[39]$} \\
\hline
\end{tabular}


(Table 2) contd...

\begin{tabular}{|c|c|c|c|}
\hline Study & $\begin{array}{l}\text { Sample Tested } \\
\text { (Plasma:P; Erythrocytes:E; Urine:U; Serum: S; Muscle } \\
\text { Extract:ME; Saliva:Sa, Breath:B; Whole Blood:WB; Capil- } \\
\text { lary Blood:CB) }\end{array}$ & $\begin{array}{l}\text { Metabolite Pathways } \\
(\downarrow \text { : reduced post- exercise, } \uparrow: \text { increased post-exercise) }\end{array}$ & $\begin{array}{l}\text { Ref. } \\
\text { No. }\end{array}$ \\
\hline Lewis, et al. & $\mathrm{P}$ & $\begin{array}{l}\text { Group } 1 \text { (pulmonary artery (PA) and superior vena cava (SVC)) } \\
\text { steep instantaneous PA-to- SVC gradients at peak exercise: } \\
\text { Purine degradation products } \\
\text { Span } 2 \text { TCA cycle intermediates } \\
\text { Differentially expressed: } \\
\text { Amino Acids (SVC \& PA) } \\
\text { Acetoacetate (SVC \& PA) } \\
\text { Glucose-6-phosphate (SVC \& PA) } \\
\text { Group 2 (P) } \\
\text { Lactate, } \uparrow \\
\text { Pyruvate, } \uparrow \\
\text { Glycerol, } \uparrow \\
\text { Alanine, } \uparrow \\
\text { Glucose-6-phosphate, } \uparrow \\
\text { Acetoacetate, } \downarrow \\
\text { Pantothenate, } \uparrow \text { in more fit individuals } \\
\text { Methionine, } \uparrow \text { in less fit individuals } \\
\text { Glutamine, } \uparrow \text { in less fit individuals } \\
\text { Group } 3 \text { (P) } \\
\text { Alanine, } \downarrow \\
\text { Threonine, } \downarrow \\
\text { Serine, } \downarrow \\
\text { Proline, } \downarrow \\
\text { Valine, } \downarrow \\
\text { Histidine, } \downarrow \\
\text { Glutamine, } \downarrow \\
\text { Asparagine, } \downarrow \\
\text { Glucose-6-phosphate, } \uparrow \\
\text { Glycerol, } \uparrow \\
\beta \text {-hydroxybutyrate, } \uparrow \\
\text { Allantoin, } \uparrow \\
\text { Kynurenate, } \uparrow \\
\text { Quinolinate, } \uparrow \\
\text { Anthranilate, } \uparrow \\
\text { Lactate, } \uparrow \\
\text { Pyruvate, } \uparrow \\
\end{array}$ & [40] \\
\hline $\begin{array}{l}\text { Pohjanen, et } \\
\text { al. }\end{array}$ & S & $\begin{array}{l}34 \text { changing metabolites [not presented in paper by authors] } \\
\text { Glycerol and Asparagine confirmed only }\end{array}$ & [41] \\
\hline Yan, et al. & S & $\begin{array}{l}\text { Alanine, } \downarrow \text { post } 2 \text { wks training Group } 2 \\
\text { Pyroglutamic acid, } \downarrow \text { post } 2 \text { wks training Group } 1 \\
\beta \text {-D-methylglucopyranoside, } \uparrow \text { post } 2 \text { wks training Group } 2 \\
\text { Valine, } \downarrow \text { post } 2 \text { wks training Group } 2 \\
\text { Uric acid, } \downarrow \text { post } 2 \text { wks training Group } 2 \\
\text { Glutamine, } \downarrow \text { post } 2 \text { wks training Group } 2 \\
\text { Glutamic acid, } \downarrow \text { post 2wks training Group } 2 \\
\text { Phenylalanine, } \downarrow \text { post } 2 \text { wks training Group } 1 \\
\text { Tyrosine, } \downarrow \text { post 2wks training Group } 1 \\
\text { Threonic acid, } \uparrow \text { post } 2 \text { wks training Group } 2 \\
\text { Ornithine, } \downarrow \text { post } 2 \text { wks training Group } 1\end{array}$ & [42] \\
\hline
\end{tabular}


(Table 2) contd....

\begin{tabular}{|c|c|c|c|}
\hline Study & $\begin{array}{l}\text { Sample Tested } \\
\text { (Plasma:P; Erythrocytes:E; Urine:U; Serum: S; Muscle } \\
\text { Extract:ME; Saliva:Sa, Breath:B; Whole Blood:WB; Capil- } \\
\text { lary Blood:CB) }\end{array}$ & $\begin{array}{l}\text { Metabolite Pathways } \\
\text { ( } \downarrow \text { : reduced post--exercise, } \uparrow: \text { increased post-exercise) }\end{array}$ & $\begin{array}{l}\text { Ref. } \\
\text { No. }\end{array}$ \\
\hline $\begin{array}{l}\text { Pechlivanis, } \\
\text { et al. }\end{array}$ & $\mathrm{U}$ & $\begin{array}{l}\text { 2-Hydroxyisovalerate, } \uparrow \\
\text { 2-Hydroxybutyrate, } \uparrow \\
\text { 2-Oxoisocaproate, } \uparrow \\
\text { 3-Hydroxyisobutyrate, } \uparrow \\
\text { 3-Methyl-2-oxovalerate, } \uparrow \\
\text { 2-Oxoisovalerate, } \uparrow \\
\text { Lactate, } \uparrow \\
\text { Alanine, } \uparrow \\
\text { Pyruvate, } \uparrow \\
\text { Citrate, } \downarrow \\
\text { 2-Oxoglutarate, } \uparrow \\
\text { Trimethylamine N-oxide, } \downarrow \\
\text { Glycine, } \downarrow \\
\text { Fumarate, } \uparrow \\
\text { Tryptophan, } \downarrow \\
\text { Hypoxanthine, } \uparrow \\
\text { Formate, } \downarrow \\
\text { Histidine }\end{array}$ & [43] \\
\hline $\begin{array}{l}\text { Pechlivanis, } \\
\text { et al. }\end{array}$ & $\mathrm{S}$ & $\begin{array}{l}\text { Elevated with training Group A: } \\
\text { 1. Methylguan } \\
\text { 2. Citrate } \\
\text { 3. Glucose } \\
\text { 4. Taurine } \\
\text { 5. Trimethylamine N-oxide } \\
\text { 6. Choline-containing compounds } \\
\text { 7. Histidines } \\
\text { 8. Acetoacetate/acetone } \\
\text { Elevated } \\
\text { 1. Acetd aining Group B: } \\
\text { 2. Methylguanidine } \\
\text { 3. Valine } \\
\text { 4. Choline-containing compounds } \\
\text { Lactate an }\end{array}$ & [44] \\
\hline $\begin{array}{l}\text { Mukherjee, } \\
\text { et al. }\end{array}$ & $\mathrm{U}$ & 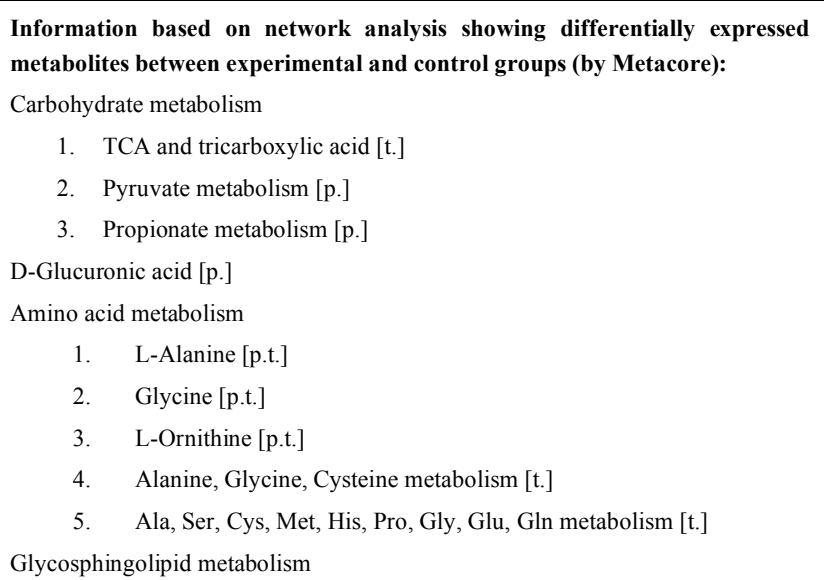 & [45] \\
\hline
\end{tabular}




\begin{tabular}{|c|c|c|c|}
\hline Study & $\begin{array}{l}\text { Sample Tested } \\
\text { (Plasma:P; Erythrocytes:E; Urine:U; Serum: S; Muscle } \\
\text { Extract:ME; Saliva:Sa, Breath:B; Whole Blood:WB; Capil- } \\
\text { lary Blood:CB) }\end{array}$ & $\begin{array}{l}\text { Metabolite Pathways } \\
\text { ( } \downarrow \text { : reduced post- exercise, } \uparrow: \text { increased post-exercise) }\end{array}$ & $\begin{array}{l}\text { Ref. } \\
\text { No. }\end{array}$ \\
\hline $\begin{array}{l}\text { Santone, et } \\
\text { al. }\end{array}$ & $\mathrm{Sa}$ & $\begin{array}{l}\text { Urea } \\
\text { Glucose } \\
\text { Lactate } \\
\text { Citrate } \\
\text { Acetate } \\
\text { Glycerol } \\
\text { Glutamate } \\
\text { Leucine } \\
\text { Alanine } \\
\text { Lysine } \\
\text { Ornithine } \\
\text { Myo-inositol } \\
\text { Glutamate } \\
\text { Creatine } \\
\text { Choline } \\
\text { Tyrosine } \\
\text { Glutamine } \\
\text { Aspartate }\end{array}$ & [46] \\
\hline $\begin{array}{l}\text { Lustgarten, } \\
\text { et al. }\end{array}$ & S & $\begin{array}{l}\text { Metabolites positively associated with } \mathrm{V}_{2} \text { max: } \\
\text { Pyridoxate } \\
\text { 2-oleoylglycerophosphoethanolamine } \\
\text { 2-hydroxyisobutyrate } \\
\text { Erythrulose } \\
\text { Tryptophan } \\
\text { Leucylleucine } \\
\text { Glycerate } \\
\text { 4-ethylphenylsulfate } \\
\text { Pantothenate } \\
\text { 4-vinylphenol ulphate } \\
\text { Erythronate } \\
\text { N-acetylornithine } \\
7 \alpha \text {-hydroxy-3-oxo-4-cholestenoate } \\
\alpha \text {-hydroxyisovalerate } \\
\text { Ergothioneine } \\
\text { Tryptophan betaine } \\
\text { Phenyllactate } \\
\text { Phenol ulphate } \\
\text { Indolelactate } \\
\text { Metabolites negatively associated with } \mathrm{V} \quad 2 \mathrm{max} \text { : } \\
\gamma \text {-tocopherol } \\
5 \alpha \text {-pregnan-3 } \beta, 20 \alpha \text {-diol disulfate } \\
\text { Stearoyl sphingomyelin } \\
\text { Pentadecanoate }\end{array}$ & [47] \\
\hline
\end{tabular}


(Table 2) contd.....

\begin{tabular}{|c|c|c|c|}
\hline Study & $\begin{array}{l}\text { Sample Tested } \\
\text { (Plasma:P; Erythrocytes:E; Urine:U; Serum: S; Muscle } \\
\text { Extract:ME; Saliva:Sa, Breath:B; Whole Blood:WB; Capil- } \\
\text { lary Blood:CB) }\end{array}$ & $\begin{array}{l}\text { Metabolite Pathways } \\
\text { ( } \downarrow \text { : reduced post- exercise, } \uparrow: \text { increased post-exercise) }\end{array}$ & $\begin{array}{l}\text { Ref. } \\
\text { No. }\end{array}$ \\
\hline Netzer, et al. & CB (earlobe) & $\begin{array}{l}\text { Increased connectivity in relation to physical activity: } \\
\text { 1. Lactate } \\
\text { 2. Alanine } \\
\text { 3. Acyl-carnitines ( } \mathrm{C} 2 \text { and } \mathrm{C} 3 \text { ) } \\
\text { Other relations: } \\
\text { Glycine }\end{array}$ & [49] \\
\hline $\begin{array}{l}\text { Lehmann, et } \\
\text { al. }\end{array}$ & $\mathrm{P}$ and $\mathrm{ME}$ & $\begin{array}{l}\text { Hlppurate, } \uparrow(\mathbf{P}) \\
\text { C6:0 carnitine, } \uparrow \text { (P) } \\
\text { C8:0 carnitine, } \uparrow(\mathbf{P}) \\
\text { C10:1 carnitine, } \uparrow \text { (P) } \\
\text { C10:0 carnitine, } \uparrow(\mathbf{P}) \\
\text { C14:2 carnitine, } \uparrow(\mathbf{P}) \\
\text { C12:0 carnitine, } \uparrow \text { (P) } \\
\text { Norepinephrine, } \uparrow \text { (P) }\end{array}$ & [50] \\
\hline
\end{tabular}


(Table 2) contd....

\begin{tabular}{|c|c|c|c|}
\hline Study & $\begin{array}{l}\text { Sample Tested } \\
\text { (Plasma:P; Erythrocytes:E; Urine:U; Serum: S; Muscle } \\
\text { Extract:ME; Saliva:Sa, Breath:B; Whole Blood:WB; Capil- } \\
\text { lary Blood:CB) }\end{array}$ & $\begin{array}{l}\text { Metabolite Pathways } \\
(\downarrow: \text { reduced post- exercise, } \uparrow: \text { increased post-exercise) }\end{array}$ & $\begin{array}{l}\text { Ref. } \\
\text { No. }\end{array}$ \\
\hline Enea, et al. & $\mathrm{U}$ & $\begin{array}{l}\text { Lactate, } \uparrow \\
\text { Alanine, } \uparrow \\
\text { Acetate, } \uparrow \\
\text { Pyruvate, } \uparrow \\
\text { Succinate, } \uparrow \\
\text { Hypoxanthine, } \uparrow\end{array}$ & [51] \\
\hline Neal, et al. & $\mathrm{U}$ (first pass) & $\begin{array}{l}\text { Hippuric acid, } \downarrow \text { post THR } \\
\text { Creatinine, } \uparrow \text { post THR } \\
\text { Dimethylamine, } \uparrow \text { post THR } \\
\text { 3-methylxanthine, } \uparrow \text { post THR } \\
\text { Hypoxanthine, } \downarrow \text { post THR }\end{array}$ & [52] \\
\hline $\begin{array}{l}\text { Nieman, et } \\
\text { al. }\end{array}$ & $P$ & $\begin{array}{l}\mathbf{8 0} \text { Total Metabolites Across: } \\
\text { Lipid metabolism } \\
\text { Carnitine metabolism } \\
\text { Ketones } \\
\text { Dicarboxylate and long chain fatty acids } \\
\text { 2. Linoleate, } \uparrow \\
\text { 3. Linolenate, } \uparrow \\
\text { 4. Dihomo-linolenate, } \uparrow \\
\text { 5. } \\
\text { Arachidonate, } \uparrow \\
\text { 7. } \\
\text { Adrenate, } \uparrow \\
\text { 8. } \quad \text { Docosapentaenoate, } \uparrow \\
\text { 9. } \quad \text { (Z)-9,10-dihydroxyoctadec-12-enoic acid and (Z)-12,13- } \\
\text { dihydroxyoctadec-9-enoic acid, } \uparrow\end{array}$ & [55] \\
\hline
\end{tabular}


(Table 2) contd....

\begin{tabular}{|c|c|c|c|}
\hline Study & $\begin{array}{l}\text { Sample Tested } \\
\text { (Plasma:P; Erythrocytes:E; Urine:U; Serum: S; Muscle } \\
\text { Extract:ME; Saliva:Sa, Breath:B; Whole Blood:WB; Capil- } \\
\text { lary Blood:CB) }\end{array}$ & $\begin{array}{l}\text { Metabolite Pathways } \\
(\downarrow: \text { reduced post--exercise, } \uparrow: \text { increased post-exercise) }\end{array}$ & $\begin{array}{l}\text { Ref. } \\
\text { No. }\end{array}$ \\
\hline $\begin{array}{l}\text { Chorell, et } \\
\text { al. }\end{array}$ & $\mathrm{P}$ & $\begin{array}{l}\text { Response to Exercise: } \\
\text { Hypoxanthine, } \uparrow \\
\text { Taurine, } \uparrow \\
\text { Ribose, } \uparrow \\
\text { Beta-D- methylglucopyranoside, } \uparrow \\
\text { Inositol, } \uparrow \\
\text { Citric acid, } \uparrow \\
\text { Beta-alanine, } \uparrow \\
\text { Malic acid, } \uparrow \\
\text { Tryptophan, } \downarrow \\
\text { Threonine, } \downarrow \\
\text { Threonate, } \downarrow \\
\text { Valine, } \downarrow \\
\text { Isoleucine, } \downarrow\end{array}$ & {$[56]$} \\
\hline $\begin{array}{l}\text { Thysell, et } \\
\text { al. }\end{array}$ & $\mathrm{S}$ & \begin{tabular}{cl}
\multicolumn{3}{c}{ Elevated post-exercise $\boldsymbol{v s}$. pre: } \\
1. & Fatty acids \\
2. & Alanine \\
3. & Inosine \\
Decrease post-exercise $\boldsymbol{v s}$. pre: \\
1. & Asparagine \\
2. & Lysine \\
3. & Serine \\
4. & Phenylalanine \\
5. & Methionine \\
6. & Arginine \\
7. & Ornithine \\
8. & Proline \\
9. & Histidine \\
10. & Allothreonine \\
11. & Tryptophan \\
12. & Valine \\
13. & Isoleucine
\end{tabular} & {$[57]$} \\
\hline Morris, et al. & $\mathrm{U}$ (second void) and $\mathrm{P}$ & $\begin{array}{l}\text { Significant group and gender interaction (U) with fitness: } \\
\text { Cystathionione } \\
\text { L-alanine } \\
\text { Glycine } \\
\text { Ethanolamine } \\
\text { L-methionine } \\
\text { L-aspartic acid } \\
\text { Homocysteine } \\
\text { Creatinine } \\
\downarrow \text { in high fitness group (P): } \\
\text { Branched chain amino acids (also in urine) } \\
\text { Leucine } \\
\text { Isoleucine } \\
\text { Valine }\end{array}$ & [58] \\
\hline
\end{tabular}


(Table 2) contd...

\begin{tabular}{|c|c|c|c|}
\hline Study & $\begin{array}{l}\text { Sample Tested } \\
\text { (Plasma:P; Erythrocytes:E; Urine:U; Serum: S; Muscle } \\
\text { Extract:ME; Saliva:Sa, Breath:B; Whole Blood:WB; Capil- } \\
\text { lary Blood:CB) }\end{array}$ & $\begin{array}{l}\text { Metabolite Pathways } \\
\text { ( } \downarrow \text { : reduced post- exercise, } \uparrow: \text { increased post-exercise) }\end{array}$ & $\begin{array}{l}\text { Ref. } \\
\text { No. }\end{array}$ \\
\hline $\begin{array}{l}\text { Sheedy, et } \\
\text { al. }\end{array}$ & U (second void) & 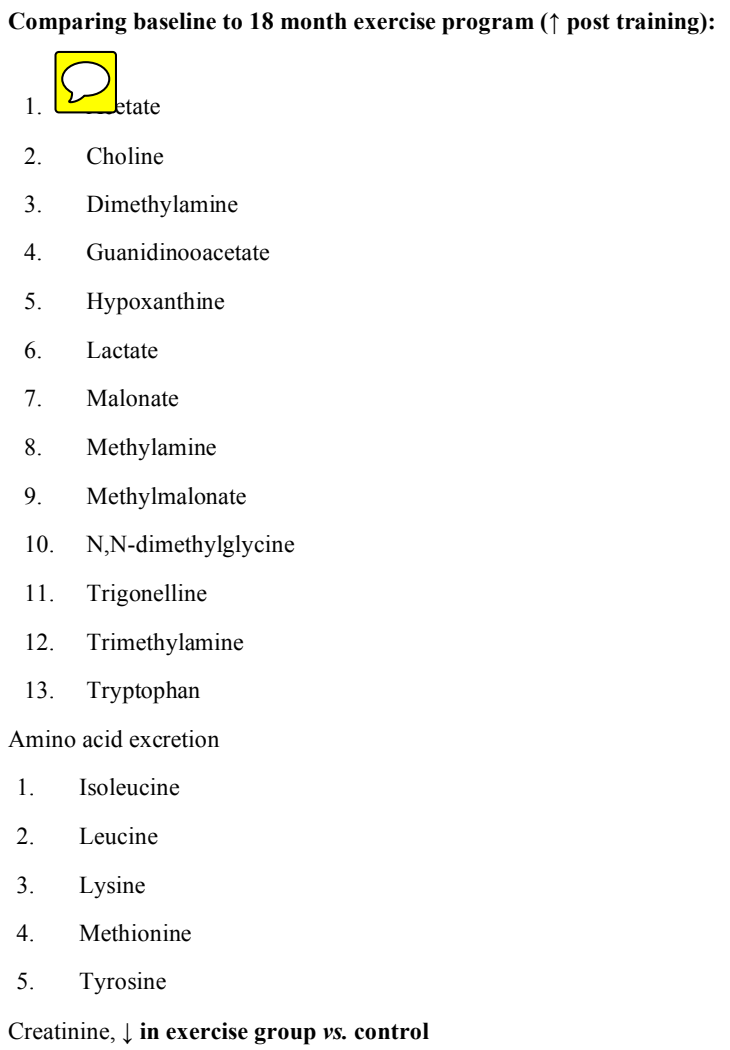 & [59] \\
\hline
\end{tabular}


(Table 2) contd....

\begin{tabular}{|c|c|c|c|}
\hline Study & $\begin{array}{l}\text { Sample Tested } \\
\text { (Plasma:P; Erythrocytes:E; Urine:U; Serum: S; Muscle } \\
\text { Extract:ME; Saliva:Sa, Breath:B; Whole Blood:WB; Capil- } \\
\text { lary Blood:CB) }\end{array}$ & $\begin{array}{l}\text { Metabolite Pathways } \\
\text { ( } \downarrow \text { : reduced post- exercise, } \uparrow: \text { increased post-exercise) }\end{array}$ & $\begin{array}{l}\text { Ref. } \\
\text { No. }\end{array}$ \\
\hline $\begin{array}{l}\text { Nieman, et } \\
\text { al. }\end{array}$ & S & $\begin{array}{l}\text { Dopamine, } \uparrow \text { post-exercise in banana supplementation group } \\
\text { Immediate response to exercise: } \\
\text { Palmitoleic Acid } \\
\text { 2,3,4-Trihydroxybutanoic Acid } \\
\text { Malic Acid } \\
\text { Succinic Acid } \\
\text { Palmitic Acid } \\
\text { Oleic Acid } \\
\text { Heptadecanoic Acid } \\
\text { D-Fructose } \\
\text { 2-Hydroxybutyric Acid } \\
\text { L-Isoleucine } \\
\text { L-Glutamic Acid } \\
\text { 2-Aminobutyric Acid } \\
\text { L-Methionine } \\
\text { Pyruvic Acid } \\
\text { L-Proline }\end{array}$ & [61] \\
\hline $\begin{array}{l}\text { Chorell, et } \\
\text { al. }\end{array}$ & S & $\begin{array}{l}\text { 3-methylhistidine, } \downarrow \text { with low } \mathrm{CHO}+\text { protein } \\
\text { Pseudouridine, } \uparrow \text { with low } \mathrm{CHO}+\text { protein } \\
\text { 4-deoxyerythronic acid, } \uparrow \text { with low CHO + protein } \\
\text { Glycine, } \downarrow \text { with low and high } \mathrm{CHO}\end{array}$ & {$[62]$} \\
\hline
\end{tabular}

Abbreviations: [t.]: transport; [p.]: pathway(s); HGPRT: hypoxanthine-guanine phosphoribosyl-transferase; ADP: adenosine 5' diphosphate; AMP: adenosine monophosphate; IMP: inosine monophosphate; ATP: adenosine 5' triphosphate; THR: threshold training distribution.

Changes in purine metabolism were monitored in longdistance runners during their yearly training cycle (preparatory, competitive and transition phases). It was found as training progressed that the levels of post- exercise xanthine decreased and the levels of the salvage enzyme HGPRT increased [16]. Observing the effect of training load and structure this time in middle-distance runners, it was proposed that plasma hypoxanthine concentration during and after exercise was affected by several factors including: the intensity of the exercise, the rate of reconversion of hypoxanthine to IMP by HGPRT, conversion of hypoxanthine to IMP by erythrocyte HGPRT, the rate of extraction by the liver and the rate of excretion. The main observation from the study was that training resulted in less excretion of hypoxanthine [17]. The same authors then compared high level sprinters and triathletes and found that hypoxanthine levels were lower in the trained sprinters in comparison with the triathletes while HGPRT levels were higher in sprinters as compared to the triathletes [18]. This suggested a more effective use of anaerobic energy resources resulting from sprint training than from triathlon training. Interestingly, in a study comparing triathletes, sprinters, middle- and long-distance runners, low hypoxanthine levels post-exercise correlated with performance to a greater extent than lactate levels and aerobic capacity [19]. Finally, these differences in purine metabolism were observed in not only elite but in amateur and recreational runners over similar 1 year training cycle. The lowest hypoxanthine levels and the highest HGPRT levels were observed in the elite runners, significant changes in purine metabolism with time were only observed after using high-intensity exercise in their training schedules, there was no significant change following constant low intensity exercise [20].

Erythrocytes are unable to synthesise purine nucleotides de novo. Measurement of adenine, guaninine nucleotides, inosine and IMP in erythrocytes post-exercise showed that AMP, ADP and IMP were decreased at 30 minutes postexercise supporting the idea that they are taken up by muscle and used to re-synthesise ATP. Hypoxanthine levels were high in red blood cells (RBC) immediately post-exercise but inosine levels were elevated at 30 minutes post-exercise indicating differences between the two metabolites in terms of rate of entry into blood [21-25].

\section{STUDIES FOCUSED ON METABOLITES RELATING TO OXIDATIVE STRESS}

Free radical production during exercise may be caused by a variety of mechanisms including a switch from xanthine dehydrogenase, which uses nicotinamide adenine nucleotide $\left(\mathrm{H}^{+}\right)$as a cofactor, to xanthine oxidase, which uses oxygen as a co-factor, and increased cyclooxygenase activity. Tocopherols and ascorbic acid protect against oxidative stress as well as compounds produced by the body such as uric acid and glutathione along with protective enzymes [26-28].

Oxidative stress in alpine skiers was evaluated after 1 day of training and 10 days of training. The markers of oxidative 
stress tocopherols, glutathione (GSH), malondialdehyde (produced from lipid oxidation), lipid peroxides and uric acid were determined. Differences in these compounds preand post-training were small [26]. The effects of extreme exercise (monitored over a two week period as part of a 6 month training programme) were assessed with regard to the antioxidant capacity of plasma. The levels of ascorbic acid, total ascorbate, and dehydroascorbate were not changed by the exercise programme but uric acid levels were increased. There was a 10 fold increase in the level of creatine phosphokinase into the plasma which is a direct indicator of exercise severity as well as a 4 fold increase in aspartate transaminase, a characteristic marker of liver injury. Plasma protein carbonyl content, a marker of protein oxidative damage, decreased significantly during exercise [27]. The levels of anti-oxidants and lipid peroxidation products were measured before and after submaximal exercise in resistance trained and non-resistance trained males. $\alpha$-tocopherol, $\gamma$-tocopherol, $\beta$-carotene, and lycopene (fat soluble antioxidants) concentrations increased after exercise. The concentrations of the lipid peroxidation products malondialdehyde and conjugated dienes were also elevated after exercise in both groups [28]. Plasma uric acid and allantoin as well as RBC malondialdehyde were measured in individuals following a short 10 minute run. It was found that uric acid and allantoin were elevated by exercise but there was no effect on plasma malondialdehyde, however, RBC malondialdehyde was elevated indicating potential lipid peroxidation at the RBC membrane during a strenuous challenge such as exercise [29].

\section{STUDIES FOCUSED ON STEROID METABOLISM}

Hydrocortisone is responsible for maintaining homeostasis within the body when it adapts to stress [30] and thus in the short term its levels may become elevated as a result of stress and give an indication of the adaptive response to stress. Cortisone is an inactive form of cortisol present in tissues which may be activated by conversion into cortisol. The levels of cortisol and cortisone were assessed post-race in elite cyclists and were found to be significantly increased post-competition [31]. Exercise was found to increase the ratio of tetrahydrocortisol to tetrahydrocortisone in urine, indicating increased generation of cortisol from cortisone at the tissue level which may be part of the adaptive response to exercise [32].

In sedentary women cortisol levels decreased following both moderate and intense exercise and returned to normal 24 hours into recovery, thus the levels determined may depend on the duration of exercise as well as when the sample was taken [33]. In addition, in both moderate and intense exercise groups, norepinephrine levels in plasma were increased post-exercise whereas epinephrine levels were only increased by intense exercise. Estradiol levels were also shown to be elevated but only by intense exercise [33]. There has also been a long standing interest in levels of plasma androgens because of their influence on the anabolic response in muscle and bone post-exercise [34]. Androgens were observed to increase in response to exercise whereas cortisol only increased after exercise. Endurance-trained subjects showed less pronounced changes in hormone concentrations in response to exercise than resistance- trained subjects. It would seem that the endogenous steroid hormone profile of men depends more on exercise mode or intensity than exercise volume as measured by caloric expenditure [35].

\section{Miscellaneous Targeted Studies}

The effects of a judo match on levels of selected plasma metabolites were determined at 3 minutes, 60 minutes and 24 hours post-match [36]. The levels of glycerol, triglycerides and free fatty acids increased 3 minutes postexercise although this might have been due to the subjects of the study having a low carbohydrate diet. Cholesterol levels were also elevated post-exercise. Serum creatinine levels rose post-exercise and this was attributed to a reduction in glomerular filtration. There was a rise in ammonia post-exercise which might be attributed to the production of xanthine and hypoxanthine but could also be attributed to protein breakdown. It was noted that levels of uric acid were elevated but not until 60 minutes after the match. At 60 minutes post- exercise the levels of glycerol, triglycerides and free fatty acids had decreased but remained higher than the resting values. This suggested that lipolysis was participating in the re-synthesis of muscular glycogen stores. Apart from uric acid and urea, the levels of the remaining markers had returned to baseline by 24 hours [36]. Furthermore, the effect of physical activity on urinary lipoxins (derived from eicosapentenoic acid) were measured and found to be elevated after an average cycle of 13.3 minutes $( \pm 2.8 \mathrm{~min})$ where subjects reached their theoretical individual heart rate maximum. These compounds are anti-inflammatory and lipoxin production may counterbalance inflammatory mediators produced by exercise [37]. The endogenous cannabinoids anandamide and 2arachindonylglycerol were measured in plasma following exercise and there were marked elevations in these compounds in both runners and cyclists post-exercise (45 min cycle at $70-80 \%$ heart rate maximum) [38]. Finally, uridine levels in blood were measured after exercise and were found to correlate with increased levels of insulin and glucose in the blood. It was proposed that the increased levels of insulin post-exercise could be due to increased secretion of catecholamines. It was found that the insulin resistance of subjects was increased post-exercise (continuous effort and progressive cycle test) and it was suggested, on the basis of a number of previous studies, that uridine has a role in insulin resistance [39].

\section{UNTARGETED OR SEMI-TARGETED ME- TABOLOMICS STUDIES}

The study by Lewis et al. [40] is by far the most comprehensive in the literature and involved around 500 subjects, although 302 of the plasma samples were pre-existing and from the Framingham Heart Study. Plasma samples were either spot samples or several blood samples were taken over a short period of time using an in-dwelling catheter. It involved targeted mass spectrometry methods so that any metabolites outside the panel of 200 selected metabolites were not monitored. The metabolites which changed following exercise highlighted the molecular pathways that might modulate the beneficial effects of exercise [40]. 
This study [40] was very detailed and the various observations are summarised below for the different exercise regimens which are detailed in tables $1_{\mathbf{\Lambda}}$ and 2. In group 2 twenty three metabolites were significantly changed at the peak exercise time. There were the expected elevations in purine metabolites, lactate and pyruvate (glycolysis), glycerol (lipolysis) as well as alanine and glutamine (gluconeogenesis). The TCA cycle intermediates malate, succinate and fumarate were elevated and the glycolysis intermediates glucose-6-phosphate and 3-phosphoglycerate were also elevated. Nicotinamide (a tryptophan metabolite) which is known to enhance insulin release was elevated in this group post-exercise. Citrulline, which is a marker of nitric oxide formation, fell post-exercise and was present to a lesser extent in the plasma of fitter individuals. Pantothenic acid, which is required for CoA biosynthesis, was elevated post-exercise and was closely correlated with fitness. Allantoin which results from the oxidation of uric acid was lower post-exercise [40].

In group 1 , the metabolic changes were similar to those in group 2 with the change being greater in the pulmonary artery compared to superior vena cava plasma suggesting that the metabolic changes derive mainly from the heart muscle or from the lower limbs. Finally, in group 3 measurements were taken after a 26.2 mile marathon and marked elevations in glycerol and $\beta$-hydroxybutyrate were consistent with extensive lipolysis and ketone body production, respectively. In contrast to the observations after acute exercise endurance exercise was followed by a reduction in the gluconeogenic amino acids alanine, threonine, serine, proline, valine, histidine, glutamine and asparagine. An unexpected observation was an increase in the tryptophan metabolites kynurenate, quinolinate, anthranilate [40].

The effect of strenuous exercise on metabolites in human serum was studied in healthy regularly trained males. Analysis was carried out by GC-TOF and followed by data extraction and multivariate statistics. Thirty four metabolites were affected by exercise although only glycerol and asparagine were reported as being fully characterised [41]. Changes in the metabolome of rowers undergoing training were investigated by using GC-TOF. Samples were taken after 1 week and 2 weeks of training. The metabolic profile of serum from professional rowers was compared with a control group. Compared with control subjects, serum of professional rowers had higher levels of alanine, lactate, cysteine, glutamic acid, valine, glutamine, and lower levels of citric acid, palmitic acid, linoleic acid and oleic acid. Alanine and tyrosine were lowered by training after both the first and second week. Pyroglutamic acid, threonic acid, glutamic acid, phenylalanine and ornithine were stable in the first week but declined after the second week. Palmitic, oleic and linoleic acid were elevated after the first week of training but returned to normal at the end of the second week. Higher levels of fatty acids in rowers compared with controls were consistent with increased energy demand as was the elevation of fatty acids after the first week of training. Alanine was higher in rowers than in the control which might be consistent with its role in the alanine-glucose cycle which promotes gluconeogenesis [42].
Human urine samples were analysed by NMR pre- and post-exercise. Five metabolites of branched chain amino acids (BCAAs) were increased post-exercise and lactate and pyruvate were greatly increased. Increases were also observed in alanine, inosine, hypoxanthine, 2-oxoglutarate and fumarate. Glycine, tryptophan, histidine, trimethylamine Noxide, citrate and formate were all decreased post-exercise. The observations on the purine metabolites reflected previous work. There was no change in the ketone bodies, acetoacetate and 3-hydroxybutyrate post-exercise and this suggested that there was no effect of exercise on the metabolism of fatty acid degradation. This as well reflects earlier work where it was observed that fat metabolism decreased with an increase in exercise intensity and there was an increase in reliance on glycogen to supply energy [43]. Additionally, it was proposed that the increase in branched-chain 2-oxo acids reflected their overproduction through transamination of BCAAs which exhibited increased turnover during exercise as a result of increased proteolysis.

An NMR- based study investigated the serum metabolic effect before and after two different training programmes (Table 1 for details). The metabolites which separated preand post-exercise were as follows: lactate and pyruvate which were highly elevated and leucine, valine, isoleucine as well as arginine/lysine which were lowered. Post 8 weeks of training the main metabolites discriminating the pre- and post-training samples were: methylguanidine, citrate, glucose, taurine, trimethylamine $\mathrm{N}$-oxide, choline-containing compounds, histidine, 1-methylhistidine, 3-methylhistidine, and acetoacetate/acetone which were all elevated and lactate, pyruvate and lipids in general were all decreased posttraining. The effect of exercise post-training was marked by increases in methylguanine and choline containing compounds and decreases in lactate and lipids. Thus the effects of training and exercise did not map strongly to particular pathways as was the case in the paper on urine by the same authors [44].

In an NMR study investigating the effects of highintensity exercise, urine samples were collected 24 hours before an exercise session and for 24 hours post. The subjects were divided into trained and untrained groups. The products of glycolysis/TCA cycle lactate, malonate and fumarate were all elevated post-exercise. As expected hypoxanthine was markedly elevated post-exercise. Acetate was elevated as well and its formation might be linked to the utilisation of pyruvate as an anti-oxidant [45]. It is likely in this study that some of the acute effects of exercise were missed through the collection of 24 hour urine samples postexercise.

NMR was used to profile metabolic changes in saliva post-exercise and link them to performance in soccer players. Tyrosine, inositol, creatine, lysine, citrate, glucose, acetate and arginine, lactate and glutamate were highlighted as being related to performance. The study indicated that salivary metabolomics using NMR was a viable technique for such studies [46]. In another study metabolomic profiling was used in order to identify markers of aerobic capacity. Blood was sampled pre-exercise and then a maximal oxygen consumption $\left(\mathrm{V} \hat{D}_{2 \max }\right)$ test was was performed. The markers which correlated most closely with $V \hat{D}_{2 \max }$ were 4- 
ethylphenylsulfate, tryptophan, $\gamma$-tocopherol, and $\alpha$ hydroxyisovalerate [47]. Elevated plasma tryptophan levels had been previously associated with lowered endurance however in this study they were positively correlated with increased endurance. Investigating the metabolite profiles (via NMR) of same sex twins where only one of the twins was physically active highlighted numerous differences. The differences between persistently physically active and inactive individuals' metabolomes indicated better cardiometabolic health in the physically active twin [48].

A targeted MS/MS approach (although methodological details were not provided) was used to monitor 60 metabolites in blood from subjects undergoing an exercise test [49]. The data was evaluated by using a computational approach and lactate, alanine, glycine and acetyl and propionyl carnitines were identified as having the strongest correlation to the effects of exercise [49]. An untargeted high resolution LC-MS method was used to examine the effect of exercise on the plasma metabolome. The major effect observed was an elevation of $\mathrm{C} 8: 0, \mathrm{C} 10: 0$ and $\mathrm{C} 12: 0$ carnitines. It was proposed that the elevation of these acyl-carnitines might be promoting beta- oxidation of palmitate through the transport of acylcarnitines into muscle cells [50].

The urinary metabolome of women divided into trained and untrained groups was examined pre- and post-exercise. The major changes were found to be in the following metabolites: lactate, pyruvate, alanine, $\beta$-hydroxybutyrate, succinate, acetate, and hypoxanthine. These metabolites were increased in both trained and untrained groups. The metabolites largely related to glycolysis and purine metabolism [51]. Two different training regimens were investigated by using NMR to profile metabolites in the urine of trained road cyclists. Their metabolic profiles did not discriminate between the training regimens. The metabolites changed following training were hippuric acid (decreased) post threshold training distribution, creatinine (increased), dimethylamine (increased) 3-methylxanthine (increased) and hypoxanthine (decreased) [52].

A targeted GC-MS metabolomic analysis was used to assess changes in tricarboxylic acid (TCA) intermediates, fatty acids, and amino acids in a comparison of the effect of high-intensity interval exercise (HIIT) and continuous moderate-intensity exercise (MOD). Twenty nine metabolites were found to change significantly after exercise. Eleven of these changed after both HIIT and MOD and thirteen only changed after HIIT. The TCA cycle intermediates citric acid, succinic acid, aconitic acid and malonic acid all increased after HIIT. Leucine and isoleucine metabolites, 4-methyl-2oxopentanoic acid and 3-methyl-2-oxopentanoic acid were increased after HIIT. Only succinic acid increased significantly after MOD. Most amino acids did not change after HIIT or MOD apart from alanine, glutamate and tyrosine, which increased after HIIT. During recovery from exercise valine, isoleucine, methionine, alanine and proline all decreased below pre-exercise levels and this was most marked following MOD. A number of fatty acids and particularly non-essential fatty acids were also elevated post-exercise [53].

The changes in the human serum metabolome produced by a 3 day period of intensified training were investigated by using GC-MS and LC-MS with compound identification based on 2800 authentic standards [54]. Immediately following the 3-day exercise period, significant 2-fold or higher increases in 75 metabolites were observed with all but twenty two of these metabolites related to lipid/carnitine metabolism. Thirteen were related to amino acid/peptide metabolism, four to hemoglobin/porphyrin metabolism, and three to Krebs cycle intermediates. After a 14 hour overnight recovery period, 50 of the 75 metabolites remained elevated. Significant decreases were observed in 22 metabolites which were primarily related to lysolipid and bile acid metabolism and all but 4 of these were still decreased after 14 hour rest recovery (details in Table 2) [54]. The multiple signatures of fatty acid beta-oxidation, omega oxidation, amino acid oxidation, and glycolysis indicated that during the 3 day exercise period the runners utilized multiple fuel types and oxidative pathways simultaneously. Novel findings from this study [54] include the influence of exercise on 12,13 hydroxyoctadec-9-(Z)-enoate (HOME) and 9-hydroxy-10,12octadedadienoic acid which were increased and are indicators or the formation of reactive oxygen species and also HOME acts as an inhibitor of neutrophil respiratory burst; enhanced omega oxidation causing an elevation in long chain dicarboxylic acids and decreases in phosphatidyl ethanolamine lipids as well as bile acids [54]. Following on from this study, oxidation products of linoleic acid were investigated in athletes after a $75 \mathrm{~K}$ cycling bout. A total of 80 metabolites had an increase of twofold or greater following exercise. The levels of several long chain fatty acids were increased in plasma and in this study, there was a particular interest in 9- and 13-hydroxyoctadecadienoic (HODE) acids. These compounds were elevated post-exercise along with several other hydroxy acids. The increased in HODES could be correlated with an increase in $F_{2}$ isoprostanes and they have potential as markers of oxidative stress during exercise [55].

GC-TOF/MS was used to characterize plasma samples from 27 individuals divided into two groups based on physical fitness level. Multi- and univariate between group comparisons based on 197 metabolites were carried out in samples collected at rest prior to any intervention and following exercise. Fitness was associated with decreased levels of $\gamma$-tocopherol and increased levels of $\alpha$-tocopherol. In addition, a high fitness level was associated with elevated levels of docosahexanoic acid and decreased levels of C18 and C16 fatty acids. Following exercise there were increases in hypoxanthine and taurine and decreases in tryptophan, threonine, valine and isoleucine [56]. The effect of exercise on metabolites in plasma from healthy subjects was monitored by using GC-TOFMS. The main changes were elevation of fatty acids and hypoxanthine post-exercise and falls in levels of amino acids including BCAAs [57].

The relationship between fitness and amino acid profile was investigated in two groups classified as being either fit or unfit. Exercise was used to classify the subjects but did not precede sampling. The group of fit subjects had lower levels of excreted amino acids and in particular higher levels of BCAAs were excreted in the unfit group. Finally, the group of fit subjects exhibited an increased rate of fat oxidation during exercise [58]. 


\section{EXERCISE STUDIES COUPLED WITH SUPPLE- MENTATION}

In healthy middle-aged males a ${ }^{1}$ HNMR study was used to examine the metabolomic changes involved with an 18 month training programme and with supplementation with calcium and vitamin D. The design was not set-up to measure the acute effects of exercise but rather measured the long term effects of an exercise programme combined with supplementation. There were no distinct changes in the urinary metabolome in response to calcium and vitamin-D3 supplementation however. Exercise produced marked changes in the urinary metabolites. Creatinine was reduced indicating perhaps greater conservation of creatinine as a result of training. Choline, dimethylamine, guanidinoacetate, hypoxanthine, lactate, malonate, methylamine, methylmalonate, N,N-dimethylglycine, trigonelline, trimethylamine and tryptophan were all elevated after the 18 months training regimen. Of particular interest was the increase in urinary choline, guanidinoacetate and hypoxanthine, in conjunction with the decrease in the urinary creatinine observed in exercisetrained participants. These metabolites point to a metabolic adaptation for utilization of anaerobically derived ATP biochemical pathways [59].

Nieman et al. [60] investigated long-distance runners that were randomly assigned to either a placebo or an intervention group. The intervention group was given water soluble polyphenols from blueberry and green tea extracts, which were captured onto a soy protein, for 17 days. For the final 3 days of the supplementation they underwent a 3 day running exercise. The main effect of the polyphenol supplementation was on levels of microbiome metabolites e.g. hippurate, 4hydroxyhippurate, 4-methylcatechol sulphate were all elevated. Fatty acid oxidation and ketogenesis were induced by exercise in both groups, with more ketones at 14 hour postexercise in the group given the supplement. Established biomarkers for inflammation and oxidative stress did not differ between the groups. The main conclusions were that the polyphenol supplementation did not alter established biomarkers for inflammation and oxidation but was linked to an enhanced gut-derived phenolic signature and ketogenesis in runners during their recovery period [60]. Another study by Nieman et al. [61] studied the effect of ingesting bananas versus a carbohydrate drink ( $\mathrm{CHO}$ ) on cycling performance and post-exercise inflammation, oxidative stress and innate immune function. Analysis of blood indicated that glucose levels and performance did not differ between the two treatments. Fifty six metabolites were affected by exercise, and only one (dopamine) had a pattern of change that differed between bananas and $\mathrm{CHO}$ and this could be attributed to the presence of dopamine in bananas. Of the top 15 metabolites changed by exercise, five were related to liver glutathione production, eight to carbohydrate, lipid, and amino acid metabolism, and two were tricarboxylic acid cycle intermediates. Banana and CHO ingestion during $75 \mathrm{~km}$ cycling resulted in similar performance, blood glucose, inflammation, oxidative stress, and innate immune levels. Thus there were no marked differences produced by the different supplements [61].

Subjects underwent four identical exercise test-sessions, including ingestion of one of four beverages (water, low- carbohydrate beverage, high carbohydrate beverage, and low-carbohydrate-protein beverage (LCHO-P) $\rightarrow$ immediately after cycling. Blood was collected at six time points, one preand five post-exercise. Extracted blood serum was analysed by GC-TOF MS. Each of the four beverages taken after exercise produced different systemic patterns. In general, a metabolic marker pattern of a catabolic state, such as increased level of fatty acids and decreased levels of amino acids and sugars, was observed to be related to the intake of water in the recovery phase. In contrast, an apparent pattern associated to an anabolic state was detected following intake of LCHO-P which increased amino acid levels and also produced an enhanced insulin response. An interesting marker of the effect of the LCHO-P drink was elevated pseudouridine which was attributed to increased RNA turnover that was a consequence of an increase in anabolic processes triggered by the availability of the amino acids in the drink and the increased insulin response it provoked. In addition, the LCHO-P drink produced reduced levels of 3methylhistidine which is a marker for myofibrillar catabolism. LCHO-P was shown to improve the metabolic status of less fit subjects in the recovery phase and the authors showed the potential for the methodology to be used for detection of early signs of insulin resistance [62].

\section{METABOLOMICS STUDIES FOCUSED ON PATIENT COHORTS}

There are ten metabolomics-based studies in the past three years which have applied some form of exercise intervention to various patient groups. The use of such technology has the potential to provide further insight into complex diseases which plague large proportions of our ever-growing human population. Yet in spite of the novel findings the crucial element will be in the application of these metabolite marker groups into treatment, prevention or other OMICS fields.

The effect of exercise and nutritional supplementation was studied in functionally limited older adults. Metabolite analysis was carried out by LC-MS and GC-MS. The regimen improved physical function and the main findings of the study reflect changes in the gut bacterial metabolism, peroxisome proliferator-activated receptor-alpha (PPAR- $\alpha$ ) activation, and insulin sensitivity. Cinnamoylglycine, phenol sulfate, p-cresol sulfate, 3-indoxyl sulfate, serotonin, hydrocinnamate, $\mathrm{N}$-methylproline, dimethylglycine, trans-urocanate, and valerate which are all believed to be gut bacterial metabolic products were all changed by the intervention and thus could be linked to improved physical function in functionally-limited older adults. $\alpha$-hydroxyisocaproate, serotonin and indolelactate are all associated with PPAR- $\alpha$ activation and were all positively associated with physical function in this study. In addition, the metabolites $\alpha$-hydroxyisocaproate, $\alpha$-hydroxyisovalerate, 2-hydroxy-3-methylvalerate, indolelactate are the reduced versions of their corresponding ketoacids which result in the consumption of nicotinamide adenine dinucleotide $(\mathrm{NADH})$ and the production of $\mathrm{NAD}^{+}$followed by an increase in the $\mathrm{NAD}^{+} / \mathrm{NADH}$ ratio that has been shown to increase PPAR- $\alpha$ expression. Finally, the metabolites myristoleate, palmitoleate; $\gamma$-glutamylglutamine, $\gamma$ glutamylalanine, $\quad \gamma$-glutamylmethionine and $\gamma$ glutamyltyrosine were positively associated with improved 
physical function and could also be linked to a lowering of insulin resistance [63].

A targeted LC-MS method and GC-MS method were used to assess the change in metabolic profile of subjects (part of the first and second STRRIDE trials) at risk from metabolic disease who either underwent a 6 month exercise programme (one of five either aerobic and/or resistance activities) or inactivity (control group). The exercise regimens with the largest energy expenditure produced marked increases in muscle concentrations of even chain acylcarnitines and these correlated positively with an increased expression of genes involved in muscle uptake and oxidation of fatty acids. The increases in acyl- carnitines and carnitine in muscle could be correlated to increases in $V D_{2 \text { peak }}$ and lowered triglyceride levels in serum. Succinic acid and succinyl carnitine had the strongest correlation to improved insulin sensitivity. Improved insulin sensitivity was also directly related to skeletal muscle concentrations of alanine, serine, methionine, glutamate/glutamine, ornithine and arginine and associations were again most pronounced in the vigorous-intensity exercise group. Surprisingly, improvement in insulin sensitivity was also related to increases in leucine and isoleucine where elevation has often been associated with insulin resistance [64]. In a previous study investigating the training-induced health benefits, Huffman et al. [65] evaluated the effect of 6 months of aerobic exercise training (3 supervised aerobic groups or inactivity) in middle-aged, sedentary, overweight to obese men and woman at risk of type 2 diabetes. Blood samples were taken at baseline, post-training and 2 weeks after training ceased. A targeted metabolomics method revealed improvements in insulin sensitivity were coupled to reductions in free fatty acids and increase in plasma glycine level that persisted 2 weeks after training completion. The study highlights the possible enhanced efficiency in mitochondrial $\beta$-oxidation return of metabolic efficiency with greater clearance of incompletely oxidised substrates from the mitochondria itself.

Pre- and post-exercise serum samples from recreationally active subjects with type 1 (T1D) diabetes and controls were analysed in serum using an ${ }^{1} \mathrm{H}-\mathrm{NMR}$ and GC-MS untargeted metabolomics approach. After the exercise (30 minutes at $80 \% \dot{V} \mathrm{O}_{2 \max }$ ), both groups had increased concentrations of alanine and lactate and TCA cycle intermediates citrate, malate, fumarate and succinate. The increase in these metabolites in T1D group was lower. The control group also showed increases in $\alpha$-ketoglutarate, $\alpha$-ketoisocaproic acid, and the lipolysis products glycerol and oleic and linoleic acids. The controls also showed a reduction in the BCAAs valine and leucine. Overall the T1D patients did not respond as strongly to exercise as the control group in terms of changes in metabolite profile, which was partly explained by the increase in serum insulin post-exercise [66].

Metabolomic profiling with NMR was carried out on serum samples from overweight women who either went on a dieting or an exercise programme for six weeks. Serum acetate and pyruvate decreased and lactate, glutamine, lactate to pyruvate ratio and fatty acids, increased in the diet group but not in the exercise group with time. Tyrosine increased in the exercise group but not in the diet group with time while phenylalanine, histidine, glycine, and $\alpha-1$-acid glyco- protein increased significantly in both groups. Glucose also decreases in the exercise group. The findings suggest that exercise was more effective at reducing risk factors associated with cardiovascular disease than dieting [67]. Moving on to chronic obstructive pulmonary disease (COPD), metabolic profiles of plasma were evaluated before and after 8 weeks of endurance exercise training in COPD patients in comparison with a control group. Samples were analyzed by ${ }^{1}$ HNMR to assess metabolite profiles. The two groups presented training-induced physiological changes in the $\dot{V} \mathrm{O}_{2 \text { peak }}$ and in blood lactate levels. Prior to endurance training COPD patients showed lower plasma levels of valine, alanine and isoleucine than healthy sedentary subjects. These amino acids showed a significant association with fat free mass index. In healthy subjects, exercise generated a significant fall in plasma level acids glutamine, tyrosine, alanine, valine, isoleucine, creatine, creatinine, citrate and glucose. As expected, exercise significantly increased plasma levels of lactate, succinate and pyruvate. The effects of exercise in COPD patients were similar to those seen in healthy subjects, except for a lack of changes in alanine and creatine in plasma. However, the responses of the COPD group were variable, and overall none of these changes were significant apart from an elevation in lactate [68].

In a previous study with NAFLD and obesity, the effects of exercise (based on guidelines issued by the Department of Health and Human Services) was investigated on weight loss as well as intrahepatic triglyceride (IHTG) content and very low density lipoprotein (VLDL) kinetics. Therefore, obese individuals with NAFLD were wither randomized into 16 weeks of exercise training $\left(45-55 \% \dot{V} \mathrm{O}_{2 \text { peak }}\right.$ for 30-60 minutes 5 days per week) or control. Blood samples were analysed via GC-MS. The exercise intervention resulted in decreased IHTG that was associated with changes seen in alanine transaminase (also decreased). Plasma free fatty acid, VLDL triglycerides and VLDL-apoB 100 were not affected by the 16 weeks of exercise neither was $\dot{V} \mathrm{O}_{2 \text { peak }}$. The findings of this study only provide very modest changes in subject metabolite profiles [69].

In a combined proteomic and metabolomics study 5 healthy lean and 5 healthy obese were subjected to a $60 \mathrm{~min}$ ute acute exercise bout (circuit training at $80 \%$ of individual maximal power). Blood samples were taken at baseline, immediately post- and 24 hours post- exercise cessation. Additionally, the authors conducted two different health intervention studies to identify possible associated biomarkers of fat mass change and glucose homeostasis. One comprised of a hypocaloric diet $(1200 \mathrm{kcal} / \mathrm{day})$ in obese patients over a period of 6 months and the second examined obese patients on a weight loss study before and after 6 months of having a gastric sleeve resection performed. Targeted serum metabolomics profiling by Biocrates Life Sciences was performed via electrospray ionisation tandem mass spectrometry identifying 163 metabolites; 12 of which significantly relate to obesity. Glycine, glutamine and glycerol- phosphatidylcholine 42:0 increased whereas glycerol- phosphatidylcholine 32:0, glycerol- phosphatidylcholine 32:1 and glycerol- phosphatidylcholine 40:5 decreased in obese patients. In response to exercise 13 metabolites in the obese and 19 in the lean group were identified. These were significantly and independent of fat mass differences. Six metabo- 
lites, in both groups, were changed and these are carnitines C2, C10:1, C14:1-OH, C14:2, C18:1 and C18:2 n. Interestingly, this study shows the improved discriminatory power when both the metabolomics and proteomics data sets are combined, which was partly owed to greater sample size however. Main changes in fat mass in response to diet and bariatric surgery were attributed to changes seen in clusterin, epithelium-derived factor, retinol binding protein 4 , iC $3 b$, glutamine and glycero- phosphatidylcholine 42:0. In terms of the pathophysiology of obesity, a down- regulation of apoptosis (sign of inflammation and oxidative stress in obesity) was a pathway associated to an up-regulation of protein abundance in obesity [70].

Metabolic health changes in insulin sensitivity, fitness, and reduced body weight were identified before and after a 14-17 week, accounting for menstrual cycle, calorie restricted diet (500-600 kcal/day reduction) and exercise intervention in sedentary, obese and insulin-resistant women. The exercise intervention comprised of 4 times weekly sessions. Over the first 4 weeks sessions were aerobic lasting for 30 minutes each on either a treadmill or cycle ergometer at 60$70 \%$ of maximal heart rate that was previously determined by a $\dot{V} \mathrm{O}_{2 \text { peak }}$ test. For weeks 5 to 8 the duration of the session was increased to 40 minutes and for the remaining weeks the intensity was increased to $75 \%$ of maximal heart rate. Subjects also performed an oral glucose tolerance test in response to the intervention. Blood samples were collected and analysed via GC/TOF-MS. Main findings in plasma fasted samples include significant increases in the 5-HMF derivatives 5-hydroxymethyl-2-furoic acid and 2, 5furandicarboxylic acid, as well as a modest but significant decrease in food-derived 1,5-anhydroglucitol (diet and microbial metabolism). There was a $40 \%$ reduction in $\alpha$ ketoglutarate, $32 \%$ lower level of the glutamate derivative gamma-aminobutyric acid and significantly reduced pyruvic acid and uric acid. Additionally a $30 \%$ reduction in behenic (C22:0) fatty acid was observed. There was also a large number of as- yet unidentified metabolites which exhibited changes as a result of the intervention. The authors also noted increased $\gamma$-tocopherol in blood post- fitness and weight loss (area under the curve) as well as stigmasterol. In fact, $\gamma$-tocopherol has been attributed to the gut microbiome and is a reflection of metabolic health. Finally, uridine decreased post- intervention which aided the discrimination of the pre- and post- time-points [71].

The effects of 6 weeks HIIT (10 times 1 minute at 90\% heart rate maximum 3 times a week) was investigated in normoglycemic yet sedentary overweight/ obese women as well as response to an oral glucose tolerance test before and after the intervention. Multi-segment injection-capillary electrophoresis- mass spectrometry was utilised to identify plasma metabolite signatures that could predict glucose tolerance changes after HIIT. Some of the main findings include, oxidized disulfides were significantly modulated after HIIT, reflected by decreased levels of cysteinylglycinecysteine disulphide, glutathionylcysteine-cysteine disulphide and cystine. Proline betaine was in contrast increased after HIIT. Other plasma metabolites which decreased significantly with time are the following: leucine, phenylalanine, methionine, isoleucine, citrulline, o-acetyl-1-carnitine, valine, lysine, tryptophan, tyrosine, histidine, ornithine (increased with training), aspartic acid as well as arginine [72].

\section{DISCUSSION}

In the untargeted studies described above some clear metabolic themes emerge, however there is also a mismatch in the consistency and volume of observations across the selected studies. This makes it difficult to order the papers according to these clear themes and at the same time indicates that the exact study design has an influence on what is being observed. For instance, the time of sampling postexercise, the type of exercise, the fitness of the participants and the analytical method utilised will all influence the markers- some to a greater or lesser extent. Therefore, in order to group these observations, generalised prominent metabolite responses have been targeted and explained below in detail.

The maximum rate of ATP re-synthesis from fatty acids is about 0.40 mol. $\mathrm{min}^{-1}$, while anaerobic breakdown of glycogen can generate from 1.0 to $2.0 \mathrm{~mol}$ of ATP $\mathrm{min}^{-1}$ [73]. Thus during high-intensity exercise, the rate of ATP breakdown is too high to be matched by the rate of ATP synthesis from fatty acids. With prolonged exercise glycogen stores become depleted and there is a greater reliance on fatty acid metabolism to supply energy [74]. Interestingly, ATP level in the resting untrained human skeletal muscle is between $20-25 \mathrm{mmol} . \mathrm{kg}^{-1}$ dry mass and this can be reduced by $20 \%$ with intense sprint training [15].

As might be expected increases in the end products of

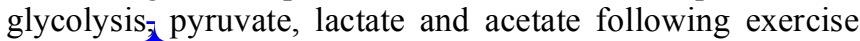
are often observed and these are more likely to increase following intense exercise and in individuals who are not trained [40, 42, 43, 45, 46, 48, 49, 51, 59, 61]. Linked to glycolysis are the metabolites within the Krebs cycle and many studies found that these metabolites were elevated post-exercise [40, 43, 44, 46, 48, 51, 53, 56, 59]. The generation of ATP from the Krebs cycle requires oxygen in the terminal respiratory chain hence during exercise there is, to a varying degree, a switch towards anaerobic glycolysis. Krebs cycle metabolites are more likely to be most elevated in trained individuals who have developed an increased aerobic capacity and who are able to oxidise pyruvate more efficiently. Another mechanism which is important in maintaining the glucose supply to muscles is gluconeogenesis, which takes place in the liver where lactate is converted to pyruvate which is then used to re-synthesise glucose. This process is energetically expensive but serves in the short term to maintain the glucose supply to muscles and thus the rapid energy supply from glycolysis can be prolonged, to some degree. It has been observed that endurance trained athletes have more efficient re-utilisation of lactate both for oxidative metabolism and gluconeogenesis [75]. Levels of alanine and glutamine were found to be elevated following exercise in several studies [43, 49, 51, 56], however, in other studies they were found to be lowered following either exercise or training $[40,55]$. Lewis et al. [40] observed elevated glutamine and alanine after acute exercise and lowered levels after endurance exercise, and it would also appear that glutamine and alanine levels fall after prolonged training [40, 42, 71]. The release of large amounts of glutamine and alanine from 
skeletal muscle post-exercise has been known for a long time $[76,77]$. Alanine and glutamine are the most important gluconeogenic amino acids and alanine is formed in the alanine cycle via conversion of lactate to pyruvate followed by transamination with glutamine as the co-factor [76]. Both glutamine and alanine are effective precursors for gluconeogenesis in the liver although the kidney also can also synthesise glucose in this case only glutamine is an effective precursor [77]. Lactate itself is used for gluconeogenesis and the advantages of releasing alanine to serve as a substrate in this pathway may be that it effectively transports both lactate and ammonia out of the muscle. Large amounts of ammonia are produced in muscle from the deamination of AMP [13] and glutamine and alanine formation might provide a mechanism for its removal. However, there is also evidence that BCAAs are the source of ammonia used for alanine and glutamine synthesis in muscle [78]. The lower level of alanine and glutamine release from muscle observed in endurance athletes is consistent with a lower requirement for gluconeogenesis resulting from a better balance between oxidative metabolism and glycolysis. However, it is also consistent with less build-up of ammonia from AMP deamination due to more efficient purine salvage in trained individuals [13-20]. In addition, in trained athletes glutamine would be required to convert IMP back to AMP and would be thus less available for pyruvate transamination or for release from the muscle to promote gluconeogenesis.

A strong theme running through many papers is the metabolism of the branched chain amino acids valine, leucine and many papers either observed decreased levels of BCAAs or increased levels of the keto- or hydroxy acids resulting from their transamination or both. Several papers observe changes in BCAAs and their metabolites [40,43,46$48,56,58,59]$. This phenomenon has been attributed to the breakdown of protein in muscles followed by metabolism of the amino acids released [43]. However, essential amino acids, particularly leucine, are also involved in stimulating biosynthesis of muscle protein $[79,80]$ so there may be two processes occurring simultaneously, oxidation of BCAAs released by protein breakdown to keto- and hydroxyacids and uptake of BCAAs from plasma for muscle repair. Hydrocortisone and testosterone metabolism control of muscle proteins and the effects of the two steroids are to some extent antagonistic with hydrocortisone promoting muscle protein catabolism [81] and testosterone promoting synthesis of muscle protein [82]. It has been observed that supplementation with BCAAs promotes the formation of muscle protein and increases plasma levels of testosterone relative to hydrocortisone [80]. As discussed above both hydrocortisone and testosterone levels have been observed to increase postexercise [32-35] so both it might be expected that both catabolism and anabolism would be observed. Other essential amino acids required for muscle protein biosynthesis such as phenylalanine, tyrosine, histidine, lysine and tryptophan have been observed to fall post-exercise as well $[40,48,71]$ and keto- and hydroxy acids derived from these amino acids have also been observed to increase [47].

Several papers observed an increase in the levels of fatty acids post-exercise $[48,53,57,58,61]$ in addition elevated glycerol has also been observed in several studies which is consistent with increased lipolysis [41, 42]. Increased lipoly- sis is consistent with the observation that hydrocortisone levels have been observed to increase post-exercise $[32,33]$ since it is widely accepted that hydrocortisone promotes the synthesis of lipases [81, 82 ] which breakdown triglycerides. Fatty acid metabolism is promoted to a greater extent in endurance athletes $[54,40]$ with depletion in glycogen reserves promoting a switch to fat metabolism and resulting in ketogenesis and oxidative metabolism. The observation of elevated fatty acid levels may again depend on exactly when post-exercise samples are taken. Romijn et al. observed that fatty acid release into plasma decreased with increasing exercise intensity [83] and fatty acid levels in plasma increased greatly during the minutes following a high-intensity exercise session. This is in line with the idea that an increase in oxidative metabolism occurs post-exercise where the NADH and flavin adenine dinucleotide produced by fatty acid $\beta$ oxidation can be used to produce ATP via the terminal respiratory chain. Tissues utilise ketone bodies (acetoacetate, $\beta$ hydroxybutyrate and acetone) as an energy source which are produced by partial metabolism of fatty acids in the liver [84]. Several studies observed an increase in ketone bodies in plasma after exercise $[40,44,58,60,61]$. Unlike glycolysis oxidative metabolism requires a supply of $\mathrm{CoA}$ and two studies observed increased levels of the CoA precursor pantothenic acid in plasma following exercise and also observed that it was positively correlated with fitness [40,47]. Pantothenate (vitamin B5) can be obtained from the diet but is also provided by intestinal bacteria. CoA is present in the mitochondria and in order for fatty acids to undergo oxidation they have to be transported into mitochondria in the form of their carnitine esters. It has been proposed that increased levels of pantothenate might indicate increased requirement for CoA but it is more likely that this indicates inhibition of CoA biosynthesis which is tightly regulated by pantothenate kinase (PK) activity [85]. Acetyl CoA inhibits PK by allosteric binding and an increase in pantothenate might indicate that in fitter individuals during exercise there is greater utilisation of oxidative metabolism via the Krebs cycle producing higher levels of acetyl CoA in comparison to individuals relying more on glycolysis for energy production. Related to observations on fatty acid metabolism there were a number of observations where acyl-carnitines were increased following exercise [47, 48, 52, 70]. The role of acyl-carnitines is ambiguous since on the one hand they transfer fatty acids into mitochondria for oxidation and on the other they remove fatty acids from the mitochondria. In addition to transferring fatty acids into the mitochondria for oxidation the acyl-carnitines promote PK activity thus increasing CoA levels in the cytosol [85]. It is more likely that the range of acyl-carnitines which are elevated following exercise is due to removal of partially oxidised fatty acids from mitochondria so that efficient oxidation of pyruvate can occur. The preferred substrates for oxidation in mitochondria are long chain acids and, for instance, Lehmann et al. [50] report that medium to short chain acyl-carnitines are elevated following exercise and speculates that they act as signalling molecules stimulating $\beta$-oxidation that but it seems more likely that they indicate removal partially oxidised acyl moieties from acyl CoAs, under conditions where the rate of glycolysis is high, in order to allow pyruvate to form acetyl CoA and enter the Krebs cycle [50]. We have observed elevated levels of acyl-carnitines in urine following exercise 
(unpublished observations) where they are certainly waste products.

Increases in the levels of gut microbial metabolites following exercise are unexpected and have been reported by four papers although not exactly the same metabolites have been reported in each case $[47,60,63,71]$. The reasons for these increases are not known although it may be as simple as an increase in blood circulation to the gut during exercise increasing the bioavailability of the products of the microbiome. This in turn might have biological effects because for instance indoxyl sulphate and cresol sulphate are uremic toxins [86]. Effects on tryptophan metabolism are also less expected and were observed by two papers which reported elevation of metabolites in the kynurenine pathway increasing following endurance exercise [40, 54]. The kyurenine pathway is a source of nicotinamide which is used for the synthesis of NADH for which there is a high demand particularly in the recovery phase of exercise where oxidative metabolism is restored. Thus this might be an explanation. However, another possibility is that mononuclear cells, which produce the tryptophan degrading enzyme indoleamine 2,3- dioxygenase, act to modulate the immune response of T-cells by depriving them of tryptophan thus preventing them from proliferating [87]. Such a response could be aimed at modulating the inflammatory response to tissue damage occurring during exercise.

\section{CONCLUSION}

The purine pathway story is by far, the most well-cited and persistent finding which dates back over 40 years. These findings highlight that the adenine nucleotide metabolites which have been shown to change according to physical fitness as well as in combination with differing training loads have been reflected in predominantly athlete and well-trained population cohorts. This could highlight a potential for the application of these markers to assess the (a) effectiveness of a given training regime in already exercising populations (b) intensity of training load based on initial resting adenine nucleotide valuations as well as, (c) the presence of overtraining/ loading in athlete groups. It would seem better to assess the acute effects of exercise rather than study the more general changes produced by training regimens over a long period since the metabolic disturbance is much greater. Thus fitness assessment and underlying health problems could be revealed by an acute exercise test and the same test could be applied after a training regimen aimed at improving health in order to evaluate the success of such a programme. Till now the observations on the effects of exercise have been rather diverse due to the reasons discussed above. By taking into consideration the scope of the analytical methods used and the optimal design for studies much greater insight into the effects of exercise will be obtained.

\section{CONFLICT OF INTEREST}

The authors confirm that this article content has no conflict of interest.

\section{ACKNOWLEDGEMENTS}

Declared none.

\section{SUPPLEMENTARY MATERIALS}

Supplementary material is available on the publisher's web site along with the published article.

\section{REFERENCES}

[1] Sarris, J.; O’Neil, A.; Coulson, C. E.; Schweitzer, I.; Berk, M. Lifestyle Medicine for Depression. BMC Psychiatry 2014, 14, 107.

[2] WHO. UNHEALTHY DIETS \& PHYSICAL INACTIVITY; 2009; pp. $1-2$.

[3] WHO $\mid$ Deaths from Cardiovascular Diseases and Diabetes.

[4] Allender, S.; Foster, C.; Scarborough, P.; Rayner, M. The Burden of Physical Activity-Related Ill Health in the UK. J. Epidemiol. Community Health 2007, 61, 344-348.

[5] WHO. Global Health Risks: Mortality and Burden of Disease Attributable to Selected Major Risks; 2009.

[6] Scarborough, P.; Bhatnagar, P.; Wickramasinghe, K. K.; Allender, S.; Foster, C.; Rayner, M. The Economic Burden of Ill Health due to Diet, Physical Inactivity, Smoking, Alcohol and Obesity in the UK: An Update to 2006-07 NHS Costs. J. Public Health (Oxf). 2011, 33, 527-535

[7] BHFNC. Economic Costs of Physical Inactivity; 2013; pp. 1-8.

[8] Reilly, J. J.; Dick, S.; McNeill, G.; Tremblay, M. S. Results From Scotland's 2013 Report Card on Physical Activity for Children and Youth. J. Phys. Act. Health 2014, 11, S93-S97.

[9] Ellis, D. I.; Dunn, W. B.; Griffin, J. L.; Allwood, J. W.; Goodacre, R. Metabolic Fingerprinting as a Diagnostic Tool Pharmacogenomics 2007, 8, 1243-1266.

[10] Nasrallah, S.; Al-Khalidi, U. Nature of Purines Excreted in Urine during Muscular Exercise. J Appl Physiol 1964, 19, 246-248.

[11] Forrester, T.; Lind, A. R. Identification of Adenosine Triphosphate in Human Plasma and the Concentration in the Venous Effluent of Forearm Muscles Before, during and after Sustained Contractions. J. Physiol. 1969, 204, 347-364.

[12] Forrester, T. The Identification and Assay of Acetylcholine and Adenosine Triphorphate Released from Active Skeletal Muscle, University of Glasgow, 1967.

[13] Sahlin, K.; Tonkonogi, M.; Söderlund, K. Plasma Hypoxanthine and Ammonia in Humans during Prolonged Exercise. Eur. J. Appl. Physiol. Occup. Physiol. 1999, 80, 417-422.

[14] Hellsten, Y.; Svensson, M.; Sjödin, B.; Smith, S.; Christensen, A.; Richter, E. .; Bangsbo, J. Allantoin Formation and Urate and Glutathione Exchange in Human Muscle during Submaximal Exercise. Free Radic. Biol. Med. 2001, 31, 1313-1322.

[15] Stathis, C. G.; Carey, M. F.; Hayes, A.; Garnham, A. P.; Snow, R. J. Sprint Training Reduces Urinary Purine Loss Following Intense Exercise in Humans. Appl. Physiol. Nutr. Metab. 2006, 31, 702708.

[16] Zieliński, J.; Rychlewski, T.; Kusy, K.; Domaszewska, K.; Laurentowska, M. The Effect of Endurance Training on Changes in Purine Metabolism: A Longitudinal Study of Competitive LongDistance Runners. Eur. J. Appl. Physiol. 2009, 106, 867-876.

[17] Zieliński, J.; Kusy, K.; Rychlewski, T. Effect of Training Load Structure on Purine Metabolism in Middle-Distance Runners. Med. Sci. Sports Exerc. 2011, 43, 1798-1807.

[18] Zielinski, J.; Kusy, K. Training-Induced Adaptation in Purine Metabolism in High-Level Sprinters vs. Triathletes. J. Appl. Physiol. 2012, 112, 542-551.

[19] Zieliński, J.; Krasińska, B.; Kusy, K. Hypoxanthine as a Predictor of Performance in Highly Trained Athletes. Int. J. Sports Med. 2013, 34, 1079-1086.

[20] Zieliński, J.; Kusy, K.; Słomińska, E. Alterations in Purine Metabolism in Middle-Aged Elite, Amateur, and Recreational Runners across a 1-Year Training Cycle. Eur. J. Appl. Physiol. 2013, 113, 763-773.

[21] Dudzinska, W.; Lubkowska, A.; Dolegowska, B.; Safranow, K.; Jakubowska, K. Adenine, Guanine and Pyridine Nucleotides in Blood during Physical Exercise and Restitution in Healthy Subjects. Eur. J. Appl. Physiol. 2010, 110, 1155-1162.

[22] Atamaniuk, J.; Vidotto, C.; Kinzlbauer, M.; Bachl, N.; Tiran, B.; Tschan, H. Cell-Free Plasma DNA and Purine Nucleotide Degradation Markers Following Weightlifting Exercise. Eur. J. Appl. Physiol. 2010, 110, 695-701. 
[23] Bianchi, G. P.; Grossi, G.; Bargossi, a M.; Fiorella, P. L.; Marchesini, G. Can Oxypurines Plasma Levels Classify the Type of Physical Exercise? J. Sports Med. Phys. Fitness 1999, 39, 123127.

[24] Gerber, T.; Borg, M. L.; Hayes, A.; Stathis, C. G. High-Intensity Intermittent Cycling Increases Purine Loss Compared with Workload-Matched Continuous Moderate Intensity Cycling. Eur. J. Appl. Physiol. 2014, 114, 1513-1520.

[25] Stathis, C. G.; Carey, M. F.; Snow, R. J. The Influence of Allopurinol on Urinary Purine Loss after Repeated Sprint Exercise in Man. Metabolism. 2005, 54, 1269-1275.

[26] Subudhi, A. W.; Davis, S. L.; Kipp, R. W.; Wayne Askew, E. Antioxidant Status and Oxidative Stress in Elite Alpine Ski Racers. Int. J. Sport. Nutr. Exerc. Metab. 2001, 11, 32-41.

[27] Chevion, S.; Moran, D. S.; Heled, Y.; Shani, Y.; Regev, G.; Abbou, B.; Berenshtein, E.; Stadtman, E. R.; Epstein, Y. Plasma Antioxidant Status and Cell Injury after Severe Physical Exercise. Proc. Natl. Acad. Sci. U. S. A. 2003, 100, 5119-5123.

[28] Ramel, A.; Wagner, K.-H.; Elmadfa, I. Plasma Antioxidants and Lipid Oxidation after Submaximal Resistance Exercise in Men. Eur. J. Nutr. 2004, 43, 2-6.

[29] Kand'ár, R.; Stramová, X.; Drábková, P.; Křenková, J. A Monitoring of Allantoin, Uric Acid, and Malondialdehyde Levels in Plasma and Erythrocytes after a 10-Minute Running Activity. Physiol. Res. 2014.

[30] De Kloet, E. R.; Karst, H.; Joëls, M. Corticosteroid Hormones in the Central Stress Response: Quick-and-Slow. Front. Neuroendocrinol. 2008, 29, 268-272.

[31] Gatti, R.; Cappellin, E.; Zecchin, B.; Antonelli, G.; Spinella, P.; Mantero, F.; De Palo, E. F. Urinary High Performance Reverse Phase Chromatography Cortisol and Cortisone Analyses before and at the End of a Race in Elite Cyclists. J. Chromatogr. B. Analyt. Technol. Biomed. Life Sci. 2005, 824, 51-56.

[32] Dovio, A.; Roveda, E.; Sciolla, C.; Montaruli, A.; Raffaelli, A.; Saba, A.; Calogiuri, G.; De Francia, S.; Borrione, P.; Salvadori, P.; Carandente, F.; Angeli, A. Intense Physical Exercise Increases Systemic 11beta-Hydroxysteroid Dehydrogenase Type 1 Activity in Healthy Adult Subjects. Eur. J. Appl. Physiol. 2010, 108, 681687.

[33] Giraldo, E.; Garcia, J. J.; Hinchado, M. D.; Ortega, E. Exercise Intensity-Dependent Changes in the Inflammatory Response in Sedentary Women: Role of Neuroendocrine Parameters in the Neutrophil Phagocytic Process and the pro-/anti-Inflammatory Cytokine Balance. Neuroimmunomodulation 2009, 16, 237-244.

[34] Pritchard, J.; Després, J.-P.; Gagnon, J.; Tchernof, A.; Nadeau, A.; Tremblay, A.; Bouchard, C. Plasma Adrenal, Gonadal, and Conjugated Steroids Following Long-Term Exercise-Induced Negative Energy Balance in Identical Twins. Metabolism 1999, 48, $1120-1127$.

[35] Tremblay, M. S.; Copeland, J. L.; Van Helder, W. Effect of Training Status and Exercise Mode on Endogenous Steroid Hormones in Men. J. Appl. Physiol. 2004, 96, 531-539.

[36] Degoutte, F.; Jouanel, P.; Filaire, E. Energy Demands during a Judo Match and Recovery. Br. J. Sports Med. 2003, 37, 245-249.

[37] Gangemi, S.; Luciotti, G.; D’Urbano, E.; Mallamace, A.; Santoro, D.; Bellinghieri, G.; Davi, G.; Romano, M. Physical Exercise Increases Urinary Excretion of Lipoxin A4 and Related Compounds. J. Appl. Physiol. 2003, 94, 2237-2240.

[38] Sparling, P. B.; Giuffrida, A.; Piomelli, D.; Rosskopf, L.; Dietrich, A. Exercise Activates the Endocannabinoid System. Neuroreport 2003, 14, 2209-2211.

[39] Dudzinska, W.; Lubkowska, a; Jakubowska, K.; Suska, M.; Skotnicka, E. Insulin Resistance Induced by Maximal Exercise Correlates with a Post-Exercise Increase in Uridine Concentration in the Blood of Healthy Young Men. Physiol. Res. 2013, 62, 163170 .

[40] Lewis, G. D.; Farrell, L.; Wood, M. J.; Martinovic, M.; Arany, Z.; Rowe, G. C.; Souza, A.; Cheng, S.; McCabe, E. L.; Yang, E.; Shi, X.; Deo, R.; Roth, F. P.; Asnani, A.; Rhee, E. P.; Systrom, D. M.; Semigran, M. J.; Vasan, R. S.; Carr, S. A.; Wang, T. J.; Sabatine, M. S.; Clish, C. B.; Gerszten, R. E. Metabolic Signatures of Exercise in Human Plasma. Sci. Transl. Med. 2010, 2,33 ra37.

[41] Pohjanen, E.; Thysell, E.; Jonsson, P.; Eklund, C.; Silfver, A.; Carlsson, I.-B.; Lundgren, K.; Moritz, T.; Svensson, M. B.; Antti, H. A Multivariate Screening Strategy for Investigating Metabolic
Effects of Strenuous Physical Exercise in Human Serum. J. Proteome Res. 2007, 6, 2113-2120.

[42] Yan, B.; A, J.; Wang, G.; Lu, H.; Huang, X.; Liu, Y.; Zha, W.; Hao, H.; Zhang, Y.; Liu, L.; Gu, S.; Huang, Q.; Zheng, Y.; Sun, J. Metabolomic Investigation into Variation of Endogenous Metabolites in Professional Athletes Subject to Strength-Endurance Training. J. Appl. Physiol. 2009, 106, 531-538.

[43] Pechlivanis, A.; Kostidis, S.; Saraslanidis, P.; Petridou, A.; Tsalis, G.; Mougios, V.; Gika, H. G.; Mikros, E.; Theodoridis, G. A. H NMR-Based Metabonomic Investigation of the Effect of Two Different Exercise Sessions on the Metabolic Fingerprint of Human Urine Research Articles. J. Proteome Res. 2010, 9, 6405-6416.

[44] Pechlivanis, A.; Kostidis, S.; Saraslanidis, P.; Petridou, A.; Tsalis, G.; Veselkov, K.; Mikros, E.; Mougios, V.; Theodoridis, G. A. 1H NMR Study on the Short- and Long-Term Impact of Two Training Programs of Sprint Running on the Metabolic Fingerprint of Human Serum. J. Proteome Res. 2013, 12, 470-480.

[45] Mukherjee, K.; Edgett, B. A.; Burrows, H. W.; Castro, C.; Griffin, J. L.; Schwertani, A. G.; Gurd, B. J.; Funk, C. D. Whole Blood Transcriptomics and Urinary Metabolomics to Define Adaptive Biochemical Pathways of High-Intensity Exercise in 50-60 Year Old Masters Athletes. PLoS One 2014, 9, e92031.

[46] Santone, C.; Dinallo, V.; Paci, M.; D’Ottavio, S.; Barbato, G.; Bernardini, S. Saliva Metabolomics by NMR for the Evaluation of Sport Performance. J. Pharm. Biomed. Anal. 2014, 88, 441-446.

[47] Lustgarten, M. S.; Price, L. L.; Logvinenko, T.; Hatzis, C.; Padukone, N.; Reo, N. V; Phillips, E. M.; Kirn, D.; Mills, J.; Fielding, R. A. Identification of Serum Analytes and Metabolites Associated with Aerobic Capacity. Eur. J. Appl. Physiol. 2013, 113, 1311-1320.

[48] Kujala, U. M.; Mäkinen, V.-P.; Heinonen, I.; Soininen, P.; Kangas, A. J.; Leskinen, T. H.; Rahkila, P.; Würtz, P.; Kovanen, V.; Cheng, S.; Sipilä, S.; Hirvensalo, M.; Telama, R.; Tammelin, T.; Savolainen, M. J.; Pouta, A.; O’Reilly, P. F.; Mäntyselkä, P.; Viikari, J.; Kähönen, M.; Lehtimäki, T.; Elliott, P.; Vanhala, M. J.; Raitakari, O. T.; Järvelin, M.-R.; Kaprio, J.; Kainulainen, H.; AlaKorpela, M. Long-Term Leisure-Time Physical Activity and Serum Metabolome. Circulation 2013, 127, 340-348.

[49] Netzer, M.; Weinberger, K. M.; Handler, M.; Seger, M.; Fang, X.; Kugler, K. G.; Graber, A.; Baumgartner, C. Profiling the Human Response to Physical Exercise: A Computational Strategy for the Identification and Kinetic Analysis of Metabolic Biomarkers. J. Clin. Bioinforma. 2011, 1, 34.

[50] Lehmann, R.; Zhao, X.; Weigert, C.; Simon, P.; Fehrenbach, E.; Fritsche, J.; Machann, J.; Schick, F.; Wang, J.; Hoene, M.; Schleicher, E. D.; Häring, H.-U.; Xu, G.; Niess, A. M. Medium Chain Acylcarnitines Dominate the Metabolite Pattern in Humans under Moderate Intensity Exercise and Support Lipid Oxidation. PLoS One 2010, 5, e11519.

[51] Enea, C.; Seguin, F.; Petitpas-Mulliez, J.; Boildieu, N.; Boisseau, N.; Delpech, N.; Diaz, V.; Eugène, M.; Dugué, B. (1)H NMRBased Metabolomics Approach for Exploring Urinary Metabolome Modifications after Acute and Chronic Physical Exercise. Anal. Bioanal. Chem. 2010, 396, 1167-1176.

[52] Neal, C. M.; Hunter, A. M.; Brennan, L.; O’Sullivan, A.; Hamilton, D. L.; De Vito, G.; Galloway, S. D. R. Six Weeks of a Polarized Training-Intensity Distribution Leads to Greater Physiological and Performance Adaptations than a Threshold Model in Trained Cyclists. J. Appl. Physiol. 2013, 114, 461-471.

[53] Peake, J. M.; Tan, S. J.; Markworth, J. F.; Broadbent, J. A.; Skinner, T. L.; Cameron-Smith, D. Metabolic and Hormonal Responses to Isoenergetic High-Intensity Interval Exercise and Continuous Moderate-Intensity Exercise. Am. J. Physiol. Endocrinol. Metab. 2014, 307, E539-E552.

[54] Nieman, D. C.; Shanely, R. A.; Gillitt, N. D.; Pappan, K. L.; Lila, M. A. Serum Metabolic Signatures Induced by a Three-Day Intensified Exercise Period Persist after $14 \mathrm{H}$ of Recovery in Runners. J. Proteome Res. 2013, 12, 4577-4584.

[55] Nieman, D. C.; Shanely, R. A.; Luo, B.; Meaney, M. P.; Dew, D. A.; Pappan, K. L. Metabolomics Approach to Assessing Plasma 13- and 9-Hydroxy-Octadecadienoic Acid and Linoleic Acid Metabolite Responses to 75-Km Cycling. AJP Regul. Integr. Comp. Physiol. 2014, 307, R68-R74.

[56] Chorell, E.; Svensson, M. B.; Moritz, T.; Antti, H. Physical Fitness Level Is Reflected by Alterations in the Human Plasma Metabolome. Mol. Biosyst. 2012, 8, 1187-1196. 
[57] Thysell, E.; Chorell, E.; Svensson, M. B.; Jonsson, P.; Antti, H. Validated and Predictive Processing of Gas Chromatography-Mass Spectrometry Based Metabolomics Data for Large Scale Screening Studies, Diagnostics and Metabolite Pattern Verification. Metabolites 2012, 2, 796-817.

[58] Morris, C.; Grada, C. O.; Ryan, M.; Roche, H. M.; De Vito, G.; Gibney, M. J.; Gibney, E. R.; Brennan, L. The Relationship between Aerobic Fitness Level and Metabolic Profiles in Healthy Adults. Mol. Nutr. Food Res. 2013, 57, 1246-1254.

[59] Sheedy, D. J. R.; Gooley, P. R.; Nahid, A.; Tull, D. L.; McConville, M. J.; Kukuljan, S.; Nowson, C. A.; Daly, R. M.; Ebeling, P. R. 1H-NMR Analysis of the Human Urinary Metabolome in Response to an 18-Month Multi-Component Exercise Program and Calcium-Vitamin-D3 Supplementation in Older Men. 2014.

[60] Nieman, D. C.; Gillitt, N. D.; Knab, A. M.; Shanely, R. A.; Pappan, K. L.; Jin, F.; Lila, M. A. Influence of a Polyphenol-Enriched Protein Powder on Exercise-Induced Inflammation and Oxidative Stress in Athletes: A Randomized Trial Using a Metabolomics Approach. PLoS One 2013, 8, e72215.

[61] Nieman, D. C.; Gillitt, N. D.; Henson, D. A.; Sha, W.; Shanely, R. A.; Knab, A. M.; Cialdella-Kam, L.; Jin, F. Bananas as an Energy Source during Exercise: A Metabolomics Approach. PLoS One 2012, 7, e37479.

[62] Chorell, E.; Moritz, T.; Branth, S.; Antti, H.; Svensson, M. B. Predictive Metabolomics Evaluation of Nutrition-Modulated Metabolic Stress Responses in Human Blood Serum During the Early Recovery Phase of Strenuous Physical Exercise Research Articles. J. Proteome Res. 2009, 8, 2966-2977.

[63] Lustgarten, M. S.; Price, L. L.; Chalé, A.; Fielding, R. A. Metabolites Related to Gut Bacterial Metabolism, Peroxisome Proliferator-Activated Receptor-Alpha Activation, and Insulin Sensitivity Are Associated with Physical Function in FunctionallyLimited Older Adults. Aging Cell 2014, 13, 918-925.

[64] Huffman, K. M.; Koves, T. R.; Hubal, M. J.; Abouassi, H.; Beri, N.; Bateman, L. A.; Stevens, R. D.; Ilkayeva, O. R.; Hoffman, E. P.; Muoio, D. M.; Kraus, W. E. Metabolite Signatures of Exercise Training in Human Skeletal Muscle Relate to Mitochondrial Remodelling and Cardiometabolic Fitness. Diabetologia 2014, 57, 2282-2295.

[65] Huffman, K. M.; Slentz, C. A.; Bateman, L. A.; Thompson, D.; Muehlbauer, M. J.; Bain, J. R.; Stevens, R. D.; Wenner, B. R.; Kraus, V. B.; Newgard, C. B.; Kraus, W. E. Exercise-Induced Changes in Metabolic Intermediates, Hormones, and Inflammatory Markers Associated with Improvements in Insulin Sensitivity. Diabetes Care 2011, 34, 174-176.

[66] Brugnara, L.; Vinaixa, M.; Murillo, S.; Samino, S.; Rodriguez, M. A.; Beltran, A.; Lerin, C.; Davison, G.; Correig, X.; Novials, A. Metabolomics Approach for Analyzing the Effects of Exercise in Subjects with Type 1 Diabetes Mellitus. PLoS One 2012, 7, e40600.

[67] Wiklund, P.; Alen, M.; Munukka, E.; Cheng, S. M.; Yu, B.; Pekkala, S.; Cheng, S. Metabolic Response to 6-Week Aerobic Exercise Training and Dieting in Previously Sedentary Overweight and Obese Pre-Menopausal Women: A Randomized Trial. J. Sport Heal. Sci. 2014, 3, 217-224.

[68] Rodríguez, D. A.; Alcarraz-Vizán, G.; Díaz-Moralli, S.; Reed, M.; Gómez, F. P.; Falciani, F.; Günther, U.; Roca, J.; Cascante, M. Plasma Metabolic Profile in COPD Patients: Effects of Exercise and Endurance Training. Metabolomics 2011, 8, 508-516.

[69] Sullivan, S.; Kirk, E. P.; Mittendorfer, B.; Patterson, B. W.; Klein, S. Randomized Trial of Exercise Effect on Intrahepatic Triglyceride Content and Lipid Kinetics in Nonalcoholic Fatty Liver Disease. Hepatology 2012, 55, 1738-1745.

[70] Oberbach, A.; Blüher, M.; Wirth, H.; Till, H.; Kovacs, P.; Kullnick, Y.; Schlichting, N.; Tomm, J. M.; Rolle-Kampczyk, U.; Murugaiyan, J.; Binder, H.; Dietrich, A.; von Bergen, M. Combined Proteomic and Metabolomic Profiling of Serum Reveals Association of the Complement System with Obesity and Identifies Novel Markers of Body Fat Mass Changes. J. Proteome Res. 2011, 10, 4769-4788.

[71] Campbell, C.; Grapov, D.; Fiehn, O.; Chandler, C. J.; Burnett, D. J.; Souza, E. C.; Casazza, G. A.; Gustafson, M. B.; Keim, N. L.; Newman, J. W.; Hunter, G. R.; Fernandez, J. R.; Garvey, W. T.; Harper, M.-E.; Hoppel, C. L.; Meissen, J. K.; Take, K.; Adams, S. H. Improved Metabolic Health Alters Host Metabolism in Parallel with Changes in Systemic Xeno-Metabolites of Gut Origin. PLoS One 2014, 9, e84260.

[72] Kuehnbaum, N. L.; Gillen, J. B.; Gibala, M. J.; Britz-McKibbin, P. Personalized Metabolomics for Predicting Glucose Tolerance Changes in Sedentary Women after High-Intensity Interval Training. Sci. Rep. 2014, 4, 6166.

[73] Zajac, A.; Poprzecki, S.; Maszczyk, A.; Czuba, M.; Michalczyk, M.; Zydek, G. The Effects of a Ketogenic Diet on Exercise Metabolism and Physical Performance in off-Road Cyclists. Nutrients 2014, 6, 2493-2508.

[74] Spriet, L. L.; Watt, M. J. Regulatory Mechanisms in the Interaction between Carbohydrate and Lipid Oxidation during Exercise. Acta Physiol. Scand. 2003, 178, 443-452.

[75] Emhoff, C.-A. W.; Messonnier, L. A.; Horning, M. A.; Fattor, J. A.; Carlson, T. J.; Brooks, G. A. Gluconeogenesis and Hepatic Glycogenolysis during Exercise at the Lactate Threshold. J. Appl. Physiol. 2013, 114, 297-306.

[76] Chang, T. W.; Goldberg, A. L. The Origin of Alanine Produced in Skeletal Muscle. J. Biol. Chem. 1978, 253, 3677-3684.

[77] Nurjhan, N.; Bucci, A.; Perriello, G.; Stumvoll, M.; Dailey, G.; Bier, D. M.; Toft, I.; Jenssen, T. G.; Gerich, J. E. Glutamine: A Major Gluconeogenic Precursor and Vehicle for Interorgan Carbon Transport in Man. J. Clin. Invest. 1995, 95, 272-277.

[78] Holecek, M. Relation between Glutamine, Branched-Chain Amino Acids, and Protein Metabolism. Nutrition 2002, 18, 130-133.

[79] Koopman, R.; Wagenmakers, A. J. M.; Manders, R. J. F.; Zorenc, A. H. G.; Senden, J. M. G.; Gorselink, M.; Keizer, H. A.; van Loon, L. J. C. Combined Ingestion of Protein and Free Leucine with Carbohydrate Increases Postexercise Muscle Protein Synthesis in Vivo in Male Subjects. Am. J. Physiol. Endocrinol. Metab. 2005, 288, E645-E653.

[80] Sharp, C. P. M.; Pearson, D. R. Amino Acid Supplements and Recovery from High-Intensity Resistance Training. J. Strength Cond. Res. 2010, 24, 1125-1130.

[81] Bergman, D. The Endocrinology of Exercise. Intern. Emerg. Med. 2013, 8 Suppl 1, S17-S21.

[82] Peckett, A. J.; Wright, D. C.; Riddell, M. C. The Effects of Glucocorticoids on Adipose Tissue Lipid Metabolism. Metabolism. 2011, 60, 1500-1510.

[83] Romijn, J. A.; Coyle, E. F.; Sidossis, L. S.; Gastaldelli, A.; Horowitz, J. F.; Endert, E.; Wolfe, R. R. Regulation of Endogenous Fat and Carbohydrate Metabolism in Relation to Exercise Intensity and Duration. Am. J. Physiol. 1993, 265, E380-E391.

[84] Fanelli, C.; Calderone, S.; Epifano, L.; De Vincenzo, A.; Modarelli, F.; Pampanelli, S.; Perriello, G.; De Feo, P.; Brunetti, P.; Gerich, J. E. Demonstration of a Critical Role for Free Fatty Acids in Mediating Counterregulatory Stimulation of Gluconeogenesis and Suppression of Glucose Utilization in Humans. J. Clin. Invest. 1993, 92, 1617-1622.

[85] Dansie, L. E.; Reeves, S.; Miller, K.; Zano, S. P.; Frank, M.; Pate, C.; Wang, J.; Jackowski, S. Physiological Roles of the Pantothenate Kinases. Biochem. Soc. Trans. 2014, 42, 1033-1036.

[86] Davila, A.-M.; Blachier, F.; Gotteland, M.; Andriamihaja, M.; Benetti, P.-H.; Sanz, Y.; Tomé, D. Intestinal Luminal Nitrogen Metabolism: Role of the Gut Microbiota and Consequences for the Host. Pharmacol. Res. 2013, 68, 95-107.

[87] Moffett, J. R.; Namboodiri, M. A. Tryptophan and the Immune Response. Immunol. Cell Biol. 2003, 81, 247-265.

[88] Baranowski, M.; Górski, J.; Klapcinska, B.; Waskiewicz, Z.; Sadowska-Krepa, E. Ultramarathon Run Markedly Reduces Plasma Sphingosine-1-Phosphate Concentration. Int. J. Sport Nutr. Exerc. Metab. 2014, 24, 148-156.

[89] Green, H. J.; Burnett, M.; Carter, S.; Jacobs, I.; Ranney, D.; Smith, I.; Tupling, S. Role of Exercise Duration on Metabolic Adaptations in Working Muscle to Short-Term Moderate-to-Heavy AerobicBased Cycle Training. Eur. J. Appl. Physiol. 2013, 113, 19651978.

[90] Green, H. J.; Burnett, M.; Jacobs, I.; Ranney, D.; Smith, I.; Tupling, S. Adaptations in Muscle Metabolic Regulation Require Only a Small Dose of Aerobic-Based Exercise. Eur. J. Appl. Physiol. 2013, 113, 313-324.

[91] Røsjø, H.; Opstad, P.-K.; Hoff, J. E.; Godang, K.; Christensen, G.; Stridsberg, M.; Omland, T. Effect of Short- and Long-Term Physical Activities on Circulating Granin Protein Levels. Regul. Pept. 2013, 185, 14-19. 
[92] Spirlandeli, A. L.; Deminice, R.; Jordao, A. A.; Preto, R. Plasma Malondialdehyde as Biomarker of Lipid Peroxidation: E Ff Ects of Acute Exercise. Int. J. Sports Med. 2014, 35, 14-18.

[93] Bye, A.; Vettukattil, R.; Aspenes, S. T.; Giskeødegård, G. F.; Gribbestad, I. S.; Wisløff, U.; Bathen, T. F. Serum Levels of Choline-Containing Compounds Are Associated with Aerobic Fitness Level: The HUNT-Study. PLoS One 2012, 7, e42330.

[94] Heyman, E.; Gamelin, F.-X.; Goekint, M.; Piscitelli, F.; Roelands, B.; Leclair, E.; Di Marzo, V.; Meeusen, R. Intense Exercise Increases Circulating Endocannabinoid and BDNF Levels in Humans--Possible Implications for Reward and Depression. Psychoneuroendocrinology 2012, 37, 844-851.

[95] Krug, S.; Kastenmüller, G.; Stückler, F.; Rist, M. J.; Skurk, T.; Sailer, M.; Raffler, J.; Römisch-Margl, W.; Adamski, J.; Prehn, C.; Frank, T.; Engel, K.-H.; Hofmann, T.; Luy, B.; Zimmermann, R.; Moritz, F.; Schmitt-Kopplin, P.; Krumsiek, J.; Kremer, W.; Huber, F.; Oeh, U.; Theis, F. J.; Szymczak, W.; Hauner, H.; Suhre, K.; Daniel, $H$. The Dynamic Range of the Human Metabolome Revealed by Challenges. FASEB J. 2012, 26, 2607-2619.

[96] Baranowski, M.; Charmas, M.; Długołęcka, B.; Górski, J. Exercise Increases Plasma Levels of Sphingoid Base-1 Phosphates in Humans. Acta Physiol. (Oxf). 2011, 203, 373-380.

[97] Green, H. J.; Bombardier, E.; Burnett, M. E.; Smith, I. C.; Tupling, S. M.; Ranney, D. A. Time-Dependent Effects of Short-Term Training on Muscle Metabolism during the Early Phase of Exercise. Am. J. Physiol. Regul. Integr. Comp. Physiol. 2009, 297, R1383-R1391.

[98] Green, H. J.; Bombardier, E.; Duhamel, T. A.; Stewart, R. D.; Tupling, A. R.; Ouyang, J. Metabolic, Enzymatic, and Transporter Responses in Human Muscle during Three Consecutive Days of
Exercise and Recovery. Am. J. Physiol. Regul. Integr. Comp. Physiol. 2008, 295, R1238-R1250.

[99] Menshikova, E. V; Ritov, V. B.; Fairfull, L.; Ferrell, R. E.; Kelley, D. E.; Goodpaster, B. H. Effects of Exercise on Mitochondrial Content and Function in Aging Human Skeletal Muscle. $J$. Gerontol. A. Biol. Sci. Med. Sci. 2006, 61, 534-540.

[100] Okura, T.; Rankinen, T.; Gagnon, J.; Lussier-Cacan, S.; Davignon, J.; Leon, A. S.; Rao, D. C.; Skinner, J. S.; Wilmore, J. H.; Bouchard, C. Effect of Regular Exercise on Homocysteine Concentrations: The HERITAGE Family Study. Eur. J. Appl. Physiol. 2006, 98, 394-401.

[101] Aguiló, A.; Tauler, P.; Fuentespina, E.; Tur, J. a; Córdova, A.; Pons, A. Antioxidant Response to Oxidative Stress Induced by Exhaustive Exercise. Physiol. Behav. 2005, 84, 1-7.

[102] Imai, H.; Hayashi, T.; Negawa, T.; Nakamura, K.; Tomida, M.; Koda, K.; Tajima, T.; Koda, Y.; Suda, K.; Era, S. Strenuous Exercise-Induced Change in Redox State of Human Serum Albumin during Intensive Kendo Training. Jpn. J. Physiol. 2002, $52,135-140$

[103] Karatzaferi, C.; Haan, A. De; Mechelen, W. Van; Sargeant, A. J. Metabolic Changes in Single Human Muscle Fibres during Brief Maximal Exercise. Exp. Physiol. 2014, 86, 411-415.

[104] Zhao, S.; Snow, R. J.; Stathis, C. G.; Febbraio, M. A.; Carey, M. F Muscle Adenine Nucleotide Metabolism during and in Recovery from Maximal Exercise in Humans. J Appl Physiol 2000, 88, 1513-1519.

[105] Green, H.; Grant, S.; Bombardier, E.; Ranney, D. Initial Aerobic Power Does Not Alter Muscle Metabolic Adaptations to ShortTerm Training. Am. J. Physiol. 1999, 277, E39-E48. 


\section{The Application of Metabolomic Profiling to the Effects of Physical Activity}

Daskalaki, Evangelia; Easton, Chris; Watson, David G

01 Mendeley User Page no.

14/2/2015 12:13

Please remove space between "post-" and "exercise"

02 Mendeley User

Page no. 15

$14 / 2 / 201512: 11$

The $\mathrm{V}$ needs a dot over it as previously and the $\mathrm{O}$ is missing.

03 Mendeley User

Page no. 15

14/2/2015 12:12

Same as above

04 Mendeley User

Page no.

21

14/2/2015 12:27

Please remove space between 80 and \%

05 Mendeley User

Page no.

14/2/2015 12:15

Please make t capital and bold the no. 1 and 2

06 Mendeley User

14/2/2015 12:10

The dot should be over the $\mathrm{V}$ not $\mathrm{O}$. 